Applied and

NISTIR 5523

Computational

Mathematics

Division

Computing and Applied Mathematics Laboratory

\title{
Phase-Field Model for Solidification of a Eutectic Alloy
}

A. A. Wheeler, G. B. McFadden and W. J. Boettinger

September 1994

QC

100

.056

N0.5523

1994
Technology Administration
U.S. DEPARTMENT OF COMMERCE
National Institute of Standards and Technology

Gaithersburg, MD 20899 



\section{NISTIR 5523}

\section{Phase-Field Model for Solidification of a Eutectic Alloy}

\section{A. A. Wheeler \\ G. B. McFadden \\ W. J. Boettinger}

U.S. DEPARTMENT OF COMMERCE

Technology Administration

National institute of Standards

and Technology

Applied and Computational Mathematics Division Computing and Applied Mathematics Laboratory

Gaithersburg, MD 20899

September 1994

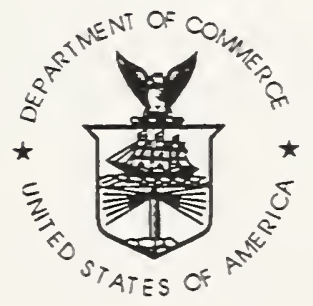

U.S. DEPARTMENT OF COMMERCE Ronald H. Brown, Secretary

TECHNOLOGY ADMINISTRATION

Mary L. Good, Under Secretary for Technology

NATIONAL INSTITUTE OF STANDARDS

AND TECHNOLOGY

Arati Prabhakar, Director 



\title{
Phase-Field Model for Solidification
}

\author{
of a Eutectic Alloy
}

\author{
A. A. Wheeler; G. B. McFadden and W. J. Boettinger \\ National Institute of Standards and Technology \\ Gaithersburg, MD 20899 USA
}

\begin{abstract}
In this paper we discuss two phase-field models for solidification of a eutectic alloy, a situation in which a liquid may transform into two distinct solid phases. The first is based on a regular solution model for the solid with a chemical miscibility gap. This model suffers from the deficiency that, in the sharp interface limit, it approximates a free-boundary problem in which the surface energy of the solid/solid interface is zero and consequently mechanical equilibrium at a trijunction requires that the solid/solid interface has zero dihedral angle (locally planar). We propose a second model which uses two order parameters to distinguish the liquid phase and the two solid phases. We provide a thermodynamically consistent derivation of this phase-field model which ensures that the local entropy production is positive. We conduct a sharp interface asymptotic analysis of the liquid/solid phase transition and show it is governed by a free-boundary problem in which both surface energy and interface kinetics are present. Finally, we consider a sharp interface analysis of a stationary trijunction between the two solid phases and the liquid phase, from which we recover the condition that the interfacial surface tensions are in mechanical equilibrium (Young's equation). This sharp interface analysis compares favourably with numerical solutions of the phase-field model appropriate to a trijunction.
\end{abstract}

*Current address: School Of Mathematics, University Walk, University of Bristol, Bristol BS8 1TW, UK. 


\section{Introduction}

Phase-field models provide an attractive framework in which to model phase transitions. Traditionally, phase transitions have been described mathematically by free-boundary problems in which the interface is represented by an evolving surface on which boundary conditions are imposed to describe the physical mechanisms occurring there. In a computational setting this formulation leads to difficulties when the interface develops a complicated geometry or when the connectedness of one of the phases changes. Such situations are commonly encountered in realistic situations where dendritic growth or coarsening occurs. As a result there are only a few computations of dendritic growth using sharp-interface methods (see, e.g., [1, 2]).

In contrast, phase-field models allow the interface between the two adjoining phases to have a non-zero width. An additional variable is introduced, the so-called phase field, whose values characterise the phase of the material at any position and time. The interface is then represented by the level sets of the phase field, which places much weaker restrictions upon the topology of the interfaces that may be computed. The governing equations for the usual thermodynamic variables, and for the phase-field, may then be derived from a Landau-Ginzburg energy functional of the system within the framework of irreversible thermodynamics $[3,4]$. The resulting set of nonlinear reaction-diffusion equations provide a coherent description of the whole system, making no distinction between the regions occupied by the different phases and the interface.

A phase-field model for a pure material was apparently first derived by Langer [5], using ideas from the study of critical phenomena [6] and other treatments in which a diffuse interface is considered $[7,8,9]$. It was subsequently studied by Caginalp $[10,11]$, and Collins and Levine [12]. In particular Caginalp [13] demonstrated the relation between phase-field and sharp interface models, showing that in different distinguished limits, in which the interface thickness is taken to zero, correspondingly different free-boundary problems are obtained. Subsequently the development of computer technology has allowed computation of numerical solutions of the phase-field equations. Kobayashi [14] and Wheeler et al. [15] have computed numerical solutions representing dendritic growth into an undercooled pure liquid. 
More recently interest has focussed on the development of phase-field models for alloy systems. Wheeler et al. [16] derived a phase-field model for isothermal solidification of a binary alloy exhibiting ideal solution behavior. Subsequently these authors [17] showed how the inclusion of a solute gradient energy in the underlying energy functional allowed a description of solute trapping in rapid solidification. Warren and Boettinger [18] extended the model of Wheeler et al. [16] to allow for different diffusivities in the solid and liquid phases and reported numerical solutions representing dendrites. Recently Caginalp [19] has developed a phase-field model for solidification of a dilute binary alloy, that includes temperature changes. Karma [20] and Elder et al. [21] have presented phase-field models for solidification of binary alloys close to the eutectic composition, where two solid phases are formed from the liquid simultaneously. Misbah and Temkin have also discussed a phase-field model of lamellar organization in the solid phase of a eutectic system [22].

In this paper we build on our previous work for isothermal solidification of alloys to derive phase-field models of eutectic alloy systems. We present two phase-field models, named I and II in sections 2 and 3 , respectively. Model I employs a regular solution model with a miscibility gap that leads to the formation of two distinct solid phases. The underlying free energy functional includes both phase-field and solute gradient energies. A model of a eutectic must be able to describe three distinct transitions: between liquid and each solid and between the two solids. The solid-solid transitions are represented by a Cahn-Hilliard equation. However, as we discuss, this approach gives, in the sharp interface limit, a free boundary problem in which the solid/solid surface energy is zero and consequently the Gibbs-Thomson effect is absent. This arises becauses the height of the double-well in the free-energy density with respect to the concentration, is not an adjustable quantity. Values for this height are routinely obtained from fitting measured phase diagram and calorimetric data by the metallurgical community; see, for example, [23]. On the other hand, the height of the double-well with respect to the phase-field can be considered to be an adjustable parameter and can be taken to be infinite in the sharp interface limit. This is done in such a manner that the surface energy of the solid/liquid interface, which is characterised by the product of the height of the double-well and the interface thickness, is finite in this limit. 
To address this difficulty we then develop Model II, which employs two independent phasefield variables to distinguish the three different phases; this approach is based upon a simpler ideal solution model for each phase. It differs from Model I in that it treats the two solid phases as arising from distinct free energies rather than a single free energy with a double-well structure. In addition to allowing a finite surface energy for the solid-solid interfaces in the sharp interface limit, this approach also provides a more general treatment of the situation in which the two solid phases have different crystalline structure and thus are not easily represented by a single free energy function. Our model II is based on a free-energy density function which has a realistic dependence on the concentration in the different phases, although the dependence on the phase field remains ad hoc. Moreover its application is not limited to dilute alloys or near-eutectic alloy systems, and hence represents a generalization of the work of Caginalp, Karma, Elder et al., and Misbah and Temkin that can describe solidification with a single model for all possible compositions. The governing equations may also be derived in a thermodynamically consistent manner, based on a entropy functional following the ideas of [3] and [4].

In section 4 we examine the sharp interface limit of our model II. For the solid/liquid interface we show that it gives a free-boundary problem in which surface energy (the Gibbs-Thomson effect) and interface kinetics are included. In section 5 we analyse a stationary trijunction in an isothermal system and show that in the sharp interface limit the interfacial surface tensions at the trijunction satisfy the usual mechanical equilibrium (Young's equation). In section 6 we present results of computations of the time independent governing equations for phase-field model II corresponding to a stationary trijunction in an imposed temperature gradient.

\section{Eutectic Phase-Field Model I}

To develop our first phase field model for a eutectic alloy we build upon our previous work $[16,17]$, in which we give a phase-field model for isothermal solidification of ideal binary alloys. In order to motivate our treatment of the eutectic system, that work is briefly reviewed in the following section. 


\subsection{The Case Of A Non-Eutectic Binary Alloy}

For this situation a phase-field model can be based on a Helmholtz free energy of the form $[16,17]$

$$
\mathcal{F}[\phi, c, T]=\int_{\mathcal{V}}\left\{f(\phi, c, T)+\frac{\epsilon_{\phi}^{2}}{2}|\nabla \phi|^{2}+\frac{\epsilon_{c}^{2}}{2}|\nabla c|^{2}\right\} d V
$$

where $\phi$ is the phase field, $c$ is the solute concentration, $T$ is the temperature, and $\mathcal{V}$ the volume of the system. The system is considered to be isothermal with solidification controlled by supersaturation rather than heat flow. Here $\phi=0$ represents the liquid and $\phi=1$ represents the solid. The bulk free energy density $f(\phi, c, T)$ is assumed to have the form appropriate to an ideal solution

$$
f(\phi, c, T)=c f_{B}(\phi, T)+(1-c) f_{A}(\phi, T)+\frac{R T}{v_{m}} I(c),
$$

where $R$ is the gas constant, $v_{m}$ is the molar volume, and

$$
I(c)=c \ln c+(1-c) \ln (1-c)
$$

and where $f_{A}$ and $f_{B}$ are the free energies of the pure components $A$ and $B$ corresponding to $c=0$ and $c=1$, respectively, and are given by

$$
f_{A}(T, \phi)=\frac{W_{A}}{4} g(\phi)+\frac{W_{A} \beta_{A}(T)}{6} h(\phi),
$$

where

$$
g(\phi)=\phi^{2}(1-\phi)^{2} \text { and } h(\phi)=\phi^{2}(3-2 \phi) .
$$

The first term $W_{A} g(\phi) / 4$ is a double-well potential, and $W_{A}$ is the barrier height between the local energy maximum at $\phi=1 / 2$ and the two minima at $\phi=0$ and $\phi=1 ; \beta_{A}(T)$ and $\beta_{B}(T)$ are taken to be linear functions of temperature whose explicit forms are given below. (Other functions for $h(\phi)$ and $g(\phi)$ could have been employed. However, in the limit of thin interfaces the result will not depend on this choice.) We note that $h(0)=0, h(1)=1$, and, since $h^{\prime}(\phi)=6 \phi(1-\phi), h(\phi)$ is increasing for $0<\phi<1$ with $h^{\prime}(0)=h^{\prime}(1)=0$. An analogous expression holds for $f_{B}(\phi, T)$. Considered as a function of $\phi$, the function $f_{A}$ has extrema at $\phi=0, \phi=1$, and $\phi=1 / 2+\beta_{A}$, and, provided $\left|\beta_{A}\right|<1 / 2$, has the form of a double-well potential with minima at $\phi=0$ and $\phi=1$ and a local maximum at $\phi=1 / 2+\beta_{A}$. 
The free energy in Eq. (2) is written as a combination of the free energies for the pure components, weighted by the solute concentrations, together with the contribution arising from the entropy of mixing term proportional to $I(c)$. This expression for the free energy can be rewritten in the equivalent form

$$
f(\phi, c, T)=h(\phi) f_{S}(c, T)+[1-h(\phi)] f_{L}(c, T)+\frac{g(\phi)}{4}\left[c W_{B}+(1-c) W_{A}\right],
$$

where $f_{S}$ and $f_{L}$ are the bulk free energies of the solid and liquid phases,

$$
\begin{gathered}
f_{S}(c, T)=\frac{R T}{v_{m}} I(c)+\left\{c \frac{W_{B} \beta_{B}(T)}{6}+(1-c) \frac{W_{A} \beta_{A}(T)}{6}\right\}, \\
f_{L}(c, T)=\frac{R T}{v_{m}} I(c) .
\end{gathered}
$$

The free energy therefore can also be written as a linear combination of the bulk free energies of the liquid and solid phases, weighted by the function $h(\phi)$, together with a contribution from a double-well potential in $\phi$ with a barrier height that varies linearly with concentration.

The time-dependent governing equations are

$$
\begin{gathered}
\frac{\partial \phi}{\partial t}=-M_{1} \frac{\delta \mathcal{F}}{\delta \phi}, \\
\frac{\partial c}{\partial t}=\nabla \cdot M_{2}\left(c(1-c) \nabla \frac{\delta \mathcal{F}}{\delta c}\right),
\end{gathered}
$$

where

$$
\frac{\delta \mathcal{F}}{\delta \phi}=\frac{\partial f}{\partial \phi}-\epsilon_{\phi}^{2} \nabla^{2} \phi
$$

and

$$
\frac{\delta \mathcal{F}}{\delta c}=\frac{\partial f}{\partial c}-\epsilon_{c}^{2} \nabla^{2} c
$$

The quantities $M_{1}$ and $M_{2}$ are positive, and may be related to the material properties of the alloy [16]. In particular

$$
M_{1}=\frac{\mu_{A} T_{A}}{6 L_{A} \delta_{A}}, \quad M_{2}=\frac{v_{m} D}{R T},
$$

where $D$ is the solute diffusivity (which for simplicity is here assumed to be the same in the solid and liquid phases), $\delta_{A}$ measures the structural thickness of the interface, and $\mu_{A}$ is the 
linear kinetic coefficient for the motion of an interface for pure A. Further, the constants $W_{A}$ and $W_{B}$, and the functions $\beta_{A}(T)$ and $\beta_{B}(T)$ may be determined from a knowledge of the surface energies, $\sigma_{A}$ and $\sigma_{B}$, the latent heats, $L_{A}$ and $L_{B}$ and the melting temperatures $T_{A}$ and $T_{B}$ of the two pure components, $A$ and $B$, through the following relations

$$
W_{A}=\frac{12 \sigma_{A}}{\delta_{A}}, \quad W_{B}=\frac{12 \sigma_{B}}{\delta_{B}},
$$

and

$$
\frac{W_{A} \beta_{A}(T)}{6}=\frac{L_{A}\left(T-T_{A}\right)}{T_{A}}, \quad \frac{W_{B} \beta_{B}(T)}{6}=\frac{L_{B}\left(T-T_{B}\right)}{T_{B}} .
$$

The parameter $\epsilon_{\phi}$ is related to the structural thickness of the interface $\epsilon_{\phi}=\sqrt{6 \sigma_{A} \delta_{A}}$. By taking the sharp interface limit, the phase-field model based upon the above energy functional reduces to a free boundary problem in which the properties of the interface, such as surface energy, are independent of the interface thickness. The remaining parameter, $\epsilon_{c}$, is related to the dependence of the alloy surface energy on composition as well as the interface adsorption [17].

\subsection{Eutectic Phase-Field Model I}

We now extend our previous phase-field model for an ideal binary alloy to a non-ideal alloy, and at the same time append a heat equation that treats non-isothermal systems. This non-ideal model will permit the treatment of a eutectic alloy whose solid phases can be represented by a single free energy function that only depends on $c$ and $T$. To this end we characterise the two solid phases, denoted by $\alpha$ and $\beta$, by their concentration and so we modify the free energy density function Eq. (2) to allow the liquid and solid phases to be regular solutions. We set

$$
\begin{aligned}
f(\phi, c, T)= & c f_{B}(\phi, T)+(1-c) f_{A}(\phi, T)+\frac{R T}{v_{m}} I(c) \\
& +\left\{h(\phi) \Omega_{S}+[1-h(\phi)] \Omega_{L}\right\} c(1-c),
\end{aligned}
$$

where $I(c)$ is given by Eq. (3), which adds to the bulk energies the terms $\Omega_{L} c(1-c)$ and $\Omega_{S} c(1-c)$ in the liquid and solid phases, respectively, and preserves the property that

$$
\left.\frac{\partial f}{\partial \phi}(\phi, c, T)\right|_{\phi=0}=\left.\frac{\partial f}{\partial \phi}(\phi, c, T)\right|_{\phi=1}=0 .
$$


The free energy can also be written in the form

$$
f(\phi, c, T)=h(\phi) f_{S}(c, T)+[1-h(\phi)] f_{L}(c, T)+\frac{g(\phi)}{4}\left[c W_{B}+(1-c) W_{A}\right],
$$

where the bulk free energies in the liquid and solid are now given by

$$
f_{L}(c, T)=\frac{R T}{v_{m}} I(c)+\Omega_{L} c(1-c),
$$

and

$$
f_{S}(c, T)=\frac{R T}{v_{m}} I(c)+\Omega_{S} c(1-c)+\left\{c \frac{L_{B}\left(T-T_{B}\right)}{T_{B}}+(1-c) \frac{L_{A}\left(T-T_{A}\right)}{T_{A}}\right\} .
$$

The bulk free energies can be used to generate a eutectic phase diagram by the appropriate choice of $T_{A}, T_{B}, L_{A}, L_{B}, \Omega_{L}$, and $\Omega_{S}$.

The governing equations are assumed to have the form

$$
\begin{gathered}
\frac{\partial \phi}{\partial t}=M_{1}\left(\epsilon^{2} \nabla^{2} \phi-\frac{\partial f}{\partial \phi}\right) \\
\frac{\partial c}{\partial t}=\nabla \cdot M_{2}\left[c(1-c) \nabla\left(\frac{\partial f}{\partial c}-\delta^{2} \nabla^{2} c\right)\right], \\
\chi \frac{\partial T}{\partial t}=k \nabla^{2} T-L^{\prime} \frac{\partial \phi}{\partial t}
\end{gathered}
$$

where we have simply adjoined an appropriate temperature equation to the other equations in the manner of previous work $[12,13,24,25]$ to treat the non-isothermal situation. The quantity $L^{\prime}$ is the alloy latent heat which we may assume to be taken as a weighted average of the latent heats of the pure components. Later we develop a more precise expression for $L^{\prime}$. For simplicity the temperature equation is written for the case of constant heat capacity $\chi$ and thermal conductivity $k$; the modifications of the model that are necessary to treat more general thermal properties are straightforward.

In order to obtain a eutectic phase diagram we require the free energy density in the solid to have a double well structure with respect to concentration, thereby providing a chemical miscibility gap. For purposes of discussion we assume a symmetric case with $T_{A}=T_{B}, W_{A}=W_{B}$ and $L_{A}=L_{B}$, in which case a double-well structure is obtained by requiring that $\Omega_{S} v_{m} / R T>2$. We assume for simplicity that $\Omega_{L}=0$, so the liquid is represented by an ideal solution. In Fig. 1 
we show a schematic diagram for the shape of the bulk free energy functions $f_{L}(c, T)$ and $f_{S}(c, T)$ of the liquid and solid phases at a given temperature.

One-dimensional steady isothermal solutions with $\phi=1$ represent stationary planar solid/solid interfaces between the $\alpha$ and $\beta$ phases. For a single, isolated interface, the concentration satisfies

$$
\mu \equiv \frac{\delta \mathcal{F}}{\delta c}=f_{c}(1, c, T)-\delta^{2} c_{z z}=\text { constant }
$$

for $-\infty<z<\infty$, where $z$ is a coordinate which measures distance from the centre of the interface, defined by the location of $c=1 / 2$; the equilibrium solute field is therefore characterised by a constant diffusion potential $\mu[26,27]$. The concentration varies with $z$ monotonically between $c_{\alpha}(T)$ and $c_{\beta}(T)$, which therefore represent the bulk concentrations in the two phases on either side of the interface. The loci $\left(c_{\alpha}(T), T\right)$ and $\left(c_{\beta}(T), T\right)$ form part of the phase diagram known as the solvus curves; they represent the solubility limit of $B$ in the $\alpha$-phase and $A$ in the $\beta$-phase, respectively. The variation of $c$ occurs on a length scale proportional to $\delta$, which therefore characterises the thickness of the solid/solid interfacial layer. The expression given by Eq. (24) has a first integral given by

$$
f(1, c, T)-\frac{\delta^{2}}{2} c_{z}^{2}=\mu c+\text { constant } .
$$

Evaluating Eqns. (24) and (25) in the bulk regions far from the interface where the gradients vanish provide the relations

$$
f_{c}\left(1, c_{\alpha}(T), T\right)=f_{c}\left(1, c_{\beta}(T), T\right)=\frac{f\left(1, c_{\alpha}(T), T\right)-f\left(1, c_{\beta}(T), T\right)}{c_{\alpha}(T)-c_{\beta}(T)}=\mu,
$$

which constitute the common tangent construction for the double-well free energy function $f(1, c, T)$. For the symmetric case under consideration, the tangent is horizontal and its slope, $\mu$, is zero. Thus, the bulk concentrations, $c_{\alpha}(T)$ and $c_{\beta}(T)$, are given by the minima of the free energy in the solid as a function of concentration. The excess Helmholtz free energy, i.e. the surface energy of the solid/solid interface, denoted by $\sigma_{S}$, is given by

$$
\sigma_{S}=\int_{-\infty}^{+\infty}\left\{\frac{\delta^{2}}{2} c_{z}^{2}+f(1, c, T)-f\left(1, c_{\alpha}(T), T\right)\right\} d z
$$


which can be manipulated by using Eq. (25) with $\mu=0$ to give

$$
\sigma_{S}=\delta \sqrt{2} \int_{c_{\alpha}(T)}^{c_{\beta}(T)} \sqrt{f(1, c, T)-f\left(1, c_{\alpha}(T), T\right)} d c .
$$

The above integral is determined entirely by the shape of the solid free energy curve as a function of concentration, and in particular depends on the value of $\Omega_{S}$, which fixes the barrier height of the double-well. The quantity $\Omega_{S}$ accounts for the regular solution nature of the free energy and is in principle available from the thermodynamic data bases for alloy systems. Thus, because $\Omega_{S}$ is finite, we see that $\sigma_{S}$ is proportional to $\delta$, and hence the interfacial width. For this model, $\sigma_{S}$ would therefore vanish in the sharp interface limit in which the interface thickness goes to zero; to maintain a finite surface energy, the interface must have finite thickness.

In contrast, we have set the problem up so that the surface energy of the solid/liquid interface does not go to zero in the corresponding sharp interface limit. The quantities $W_{A}$ and $W_{B}$ which determine the height of the double-well in the free energy with respect to $\phi$ are not available from a thermodynamic data base, and the dependence of the free energy density on $\phi$, unlike its dependence on $c$ alone, may be considered ad hoc. For this reason, in taking the sharp interface limit for the solid/liquid interface we may allow the quantities $W_{A}$ and $W_{B}$ to be inversely proportional to the interface width, so ensuring that the surface energy, which is proportional to the product of the double-well height and interface width, remains non-zero in this limit. As a consequence, the surface energy of the solid/liquid interface may be set to the appropriate physical value independent of the interface thickness.

If we take the limit in which both the thicknesses of the solid/solid and solid/liquid interfaces go to zero simultaneously, then at leading order the surface energy of the solid/solid interface vanishes, while the solid/liquid surface energies are finite. As a consequence the condition for thermodynamic equilibrium at a solid/solid interface that is recovered in this limit is independent of the local curvature at leading order, and therefore does not include the Gibbs-Thomson effect. Another unfortunate consequence of Model $I$ is that for equilibrium at a trijunction between the $\alpha, \beta$ and liquid phases, the dihedral angle is $180^{\circ}$ in the sharp interface limit; that is, the solid/liquid interface is macroscopically planar. This solution in the sharp limit cannot describe physically realistic geometries with dihedral angles other than $180^{\circ}$. 
The asymptotic analyses for the solid/solid and solid/liquid interfaces discussed above are different distinguished sharp interface limits, representing different interpretations of models of phase-field type. The approach for the solid with a chemical miscibility gap in which the height of the double-well is fixed attributes a small but finite thickness to the interface, which represents the actual physical thickness on the length scale of several interatomic distances. Such an approach is appropriate to solid/solid interfaces such as occur during spinodal decomposition, modeled by the Cahn-Hilliard equation, in which the two phases may be characterised by a quantity that is measurable in principle, namely the concentration that varies across the thin interfacial layers, see $[28,29,30]$.

The approach we have adopted for solid/liquid interfaces, in which the height of the doublewell is allowed to depend in a manner inversely proportional to the interface width, presumes that on the length scale associated with microstructure of an interface, the thickness of the interface is negligibly small. Despite its zero thickness the interface still retains a finite surface energy. This approach is appropriate to solid/liquid interfaces when the interface is characterised by a phase-field, which is not a directly observable quantity, but a construct of the model. Because of the ad hoc nature of the double-well structure of the free-energy functionals employed between liquid and solid, the validity of this approach lies in its ability to recover classical free boundary problems in the sharp interface limit. This ensures that the correct physical mechanisms are accounted for as the interface thickness is successively reduced. Thus, employing a small, finite interface thickness in a computational setting provides only a correspondingly small perturbation of the computed solution from that of the underlying classical free-boundary problem. This property, as discussed above, does not hold for the phase-field model given in this section.

The derivation of this model based on a Helmholtz free energy is another potential issue. As pointed out by Penrose and Fife ([3], see also [4]), for a non-isothermal system the entropy provides a more appropriate thermodynamic potential as a basis for deriving the phase-field equations.

The above eutectic model is quite simple. Indeed, if the free energy function is expanded about the eutectic composition to remove the logarithmic terms, it reduces to the models pro- 
posed by Karma [20] and Elder et al. [21]. It can be used to treat a solid composed of two phases that are structurally identical (the same crystal structure) and distinguishable only by their compositions. However, many eutectic systems involve a liquid in equilibrium with two solid phases that are structurally dissimilar, with distinguished crystal structures. For example, in $\mathrm{Pb}$ - $\mathrm{Sn}$ eutectic systems, the $\mathrm{Pb}$-rich $\alpha$-phase is cubic, and the $\mathrm{Sn}$-rich $\beta$-phase has tetragonal structure. The description of such systems by a simple double-well free energy function in the solid is unrealistic, since the concentration alone is not enough to uniquely characterise the phase. This leads us to consider the use of a second phase field that represents transitions between the two solid phases. Indeed this is just as reasonable as using a phase field to represent the change between liquid and solid. We thus propose a second eutectic model using a pair of phase field variables that will treat the more general eutectic system with separate free energy functions for the $\alpha$ and $\beta$ phases, as indicated schematically in Fig. 2. This second model will also permit passage to the sharp interface limit in a manner that yields non-zero surface energies for liquid-solid and solid-solid interfaces.

\section{Eutectic Phase-Field Model II}

In this section we introduce an alternative phase-field model of a eutectic that remedies the difficulties found in the previous phase-field model. We distinguish the two different solid phases by employing an additional phase field, denoted by $\psi$, so that $\psi=1$ corresponds to the $\alpha$ phase and $\psi=0$ corresponds to the $\beta$ phase. The free energy density of each phase is assumed to be that of an ideal solution. We first show how the Helmholtz free energies of the $\alpha$-solid/liquid and $\beta$-solid/liquid pairs (henceforth denoted $\alpha-\mathrm{L}$ and $\beta$-L) may be used to construct a Helmholtz free energy for a eutectic alloy. We then extend this idea to construct an entropy functional and the associated thermodynamic potentials to derive the governing phase-field equations in a thermodynamically-consistent manner for a nonisothermal system.

The governing equations for the two phase fields and for the concentration and temperature of a non-isothermal alloy are derived from an entropy functional in an analogous way to that discussed by Wang et al. [4] for the case of a pure material. The thermodynamic potentials 
used in this procedure are the internal energy, the entropy, and the Helmholtz free energy. The appropriate potentials for the eutectic model are assembled by combining basic potentials for single-component, single-phase systems. The potentials for two-phase single-component systems are obtained as above by assuming linear combinations of each phase that are weighted by the phase-field variables, augmented by a term representing a double-well potential in the phase field.

More specifically, the bulk Helmholtz free energy density for the eutectic system is written in the form

$$
f(T, c, \phi, \psi)=h(\psi) f^{\alpha L}(T, c, \phi)+[1-h(\psi)] f^{\beta L}(T, c, \phi)+\frac{W_{F \psi}}{4} h(\phi) \psi^{2}(1-\psi)^{2},
$$

where $f^{\alpha L}$ and $f^{\beta L}$ are ideal-solution free energy densities as constructed above corresponding to the $\alpha-\mathrm{L}$ and $\beta-\mathrm{I}$ phase transitions. The double-well potential in the $\psi$ field is cut off in the bulk liquid phase by the function $h(\phi)$ which for $\phi=0$ gives $h(\phi)=0$. The $\alpha-\mathrm{L}$ and $\beta-\mathrm{L}$ equilibria are then governed by separate lens-shaped phase diagrams that depend on the melting points $T_{A}^{\alpha L}, T_{B}^{\alpha L}, T_{A}^{\beta L}$, and $T_{B}^{\beta L}$, on the latent heats $L_{A}^{\alpha L}, L_{B}^{\alpha L}, L_{A}^{\beta L}$, and $L_{B}^{\beta L}$, and on the barrier heights $W_{A}^{\alpha L}, W_{B}^{\alpha L}, W_{A}^{\beta L}$, and $W_{B}^{\beta L}$. A schematic diagram illustrating the notation is given in Fig. 3. The eutectic liquid composition that can be in equilibrium with both the $\alpha$ and $\beta$ solid phases is given by the intersection of the two liquidus curves.

The definition of the Helmholtz free energy density is

$$
f=e-T s
$$

where $e$ and $s$ are the internal energy density and entropy density, respectively. The differential of the internal energy, extended to allow for dependence of the internal energy density on the phase fields, has the form

$$
d e=T d s+\mu d c+\left.\frac{\partial e}{\partial \phi}\right|_{s, c, \psi} d \phi+\left.\frac{\partial e}{\partial \psi}\right|_{s, c, \phi} d \psi
$$

it follows that

$$
d f=-s d T+\mu d c+\left.\frac{\partial e}{\partial \phi}\right|_{s, c, \psi} d \phi+\left.\frac{\partial e}{\partial \psi}\right|_{s, c, \phi} d \psi
$$


where

$$
\mu=\left.\frac{\partial f}{\partial c}\right|_{T, \phi, \psi}=\left.\frac{\partial e}{\partial c}\right|_{s, \phi, \psi}
$$

is the appropriate expression for diffusion potential in this setting. An expression for the entropy is given by

$$
s=-\left.\frac{\partial f}{\partial T}\right|_{c, \phi, \psi} .
$$

Examination of the differentials shows that the natural choice of the arguments for the thermodynamic potentials is given by $e=e(s, c, \phi, \psi), s=s(e, c, \phi, \psi)$, and $f=f(T, c, \phi, \psi)$. Before discussing the explicit forms that we consider for the thermodynamic potentials $f(T, c, \phi, \psi)$, $e(s, c, \phi, \psi)$, and $s(e, c, \phi, \psi)$, we proceed with a derivation of the governing equations. In the derivation we generalize the treatment of Wang et al. by including a gradient energy coefficient and a double-well structure for the internal energy as well as for the entropy. Following the formal derivation, however, we simplify the resulting equations to a more conventional form by assuming values for the free energy barrier height and gradient energy coefficient that are independent of temperature, and by neglecting the analogous contributions from the internal energy.

The total entropy $\mathcal{S}$, the total internal energy $\mathcal{E}$, and the total Helmholtz free energy $\mathcal{F}$ of a subvolume $V$ of the system are assumed to be of the form

$$
\begin{aligned}
& \mathcal{S}=\int_{V}\left[s(e, c, \phi, \psi)-\frac{\epsilon_{S \phi}^{2}}{2}|\nabla \phi|^{2}-\frac{\epsilon_{S \psi}^{2}}{2}|\nabla \psi|^{2}\right] d V, \\
& \mathcal{E}=\int_{V}\left[e(s, c, \phi, \psi)+\frac{\epsilon_{E \phi}^{2}}{2}|\nabla \phi|^{2}+\frac{\epsilon_{E \psi}^{2}}{2}|\nabla \psi|^{2}\right] d V, \\
& \mathcal{F}=\int_{V}\left[f(T, c, \phi, \psi)+\frac{\epsilon_{F \phi}^{2}}{2}|\nabla \phi|^{2}+\frac{\epsilon_{F \psi}^{2}}{2}|\nabla \psi|^{2}\right] d V,
\end{aligned}
$$

where the square gradient contributions from the $\phi$ and $\psi$ phase fields incorporate the effect of spatial gradients on the system as in the Cahn-Allen equation. A more general formulation could include contributions due to solute gradients as well, as in the Cahn-Hilliard equations; such terms have been employed in phase-field models of solute trapping at rapid solidification rates [17]. Here we limit our attention to slow growth conditions, a situation in which they do 
not make a significant contribution. Consistent with the thermodynamic relation $f=e-T s$, we assume that the square gradient coefficients are related by

$$
\epsilon_{F \phi}^{2}=\epsilon_{E \phi}^{2}+T \epsilon_{S \phi}^{2}, \quad \epsilon_{F \psi}^{2}=\epsilon_{E \psi}^{2}+T \epsilon_{S \psi}^{2}
$$

where we have taken into account the sign convention used in the entropy functional. In a more general treatment, surface tension anisotropy can be modeled by replacing the gradient energy coefficients by appropriate functions of the gradients of the phase fields that indicate the local orientation of the interface [31].

We next postulate governing equations which are consistent with positive local entropy production as well as the conservation of heat and solute. The fundamental balances are written

$$
\begin{gathered}
\frac{d \mathcal{S}}{d t}+\int_{\delta V} \mathrm{~J}_{\mathbf{S}} \cdot \widehat{\mathbf{n}} d A=\int_{V} S_{P} d V \geq 0 \\
\frac{d \mathcal{E}}{d t}+\int_{\delta V} \mathbf{J}_{\mathbf{e}} \cdot \widehat{\mathbf{n}} d A=0 \\
\frac{d}{d t} \int_{V} c d V+\int_{\delta V} \mathbf{J}_{\mathbf{c}} \cdot \widehat{\mathbf{n}} d A=0
\end{gathered}
$$

where $\mathbf{J}_{\mathbf{s}}, \mathbf{J}_{\mathbf{e}}$, and $\mathbf{J}_{\mathbf{c}}$ are fluxes of entropy, internal energy, and solute, respectively, and $S_{P}$ is the entropy production per unit volume. The energy and entropy fluxes are written in the form

$$
\begin{gathered}
\mathrm{J}_{\mathrm{e}}=\mathrm{q}-\epsilon_{E \phi}^{2} \phi_{t} \nabla \phi-\epsilon_{E \psi}^{2} \psi_{t} \nabla \psi, \\
\mathrm{J}_{\mathbf{s}}=\frac{1}{T} \mathrm{q}-\frac{\mu}{T} \mathrm{~J}_{\mathrm{c}}+\epsilon_{S \phi}^{2} \phi_{t} \nabla \phi+\epsilon_{S \psi}^{2} \psi_{t} \nabla \psi,
\end{gathered}
$$

where $\mathrm{q}$ is the heat flux appropriate to a single-phase system. The terms proportional to the time derivatives of the phase fields in these expressions represent fluxes related to changes to the the phase fields at the boundary of $V$ as discussed in [4]. Conservation of energy then has the form

$$
e_{t}+\nabla \cdot \mathrm{q}=\epsilon_{E \phi}^{2} \phi_{t} \nabla^{2} \phi+\epsilon_{E \psi}^{2} \psi_{t} \nabla^{2} \psi
$$

and conservation of solute gives

$$
c_{t}+\nabla \cdot \mathrm{J}_{\mathrm{c}}=0
$$


From the expressions (31) and (32), the time derivative of the entropy density can be written

$$
\begin{gathered}
\frac{\partial}{\partial t} s(e, c, \phi, \psi)=\left.\frac{\partial s}{\partial e}\right|_{c, \phi, \psi} e_{t}+\left.\frac{\partial s}{\partial c}\right|_{c, \phi, \psi} c_{t}+\left.\frac{\partial s}{\partial \phi}\right|_{e, c, \psi} \phi_{t}+\left.\frac{\partial s}{\partial \psi}\right|_{e, c, \phi} \psi_{t} \\
=\frac{1}{T} e_{t}-\frac{\mu}{T} c_{t}-\left.\frac{1}{T} \frac{\partial f}{\partial \phi}\right|_{T, c, \psi} \phi_{t}-\left.\frac{1}{T} \frac{\partial f}{\partial \psi}\right|_{T, c, \phi} \psi_{t} .
\end{gathered}
$$

By using the equations for conservation of energy and solute, together with the expression (43) for the entropy flux, the local entropy production $S_{P}$ in Eq. (39) is then found to have the form

$$
\begin{gathered}
S_{P}=\mathbf{q} \cdot \nabla\left(\frac{1}{T}\right)-\mathbf{J}_{\mathbf{c}} \cdot \nabla\left(\frac{\mu}{T}\right) \\
+\frac{\phi_{t}}{T}\left(T \epsilon_{S \phi}^{2} \nabla^{2} \phi-\left.\frac{\partial f}{\partial \phi}\right|_{T, c, \psi}\right)+\frac{\psi_{t}}{T}\left(T \epsilon_{S \psi}^{2} \nabla^{2} \psi-\left.\frac{\partial f}{\partial \psi}\right|_{T, c, \psi}\right) .
\end{gathered}
$$

Positive local entropy production is therefore guaranteed if we take

$$
\begin{gathered}
\mathrm{q}=M_{T} \nabla\left(\frac{1}{T}\right), \\
\mathrm{J}_{\mathrm{c}}=-M_{\mathrm{c}} \nabla\left(\frac{\mu}{T}\right),
\end{gathered}
$$

as well as the equations

$$
\begin{aligned}
& \phi_{t}=M_{\phi}\left[\epsilon_{F \phi}^{2} \nabla^{2} \phi-f_{\phi}\right], \\
& \psi_{t}=M_{\psi}\left[\epsilon_{F \psi}^{2} \nabla^{2} \psi-f_{\psi}\right],
\end{aligned}
$$

where $M_{c}, M_{T}, M_{\phi}$, and $M_{\psi}$ are positive and are generally functions of $\phi, \psi, c$ and $T$. As we show below, $M_{T}$ and $M_{c}$ are related to the diffusion of internal energy and solute. Crosscoupling between the heat and mass transfer could be incorporated at this stage (Soret and Dufour effects), but for simplicity we assume these effects are negligible.

In order to recover linear diffusion of heat and solute in the bulk phases we now assume that $M_{T}=\lambda T^{2}$ and $M_{c}=v_{m} D c(1-c) / R$, where $\lambda$ is the thermal conductivity and $D$ is the solute diffusivity. For simplicity, both $\lambda$ and $D$ are assumed to be constant; it is straightforward to allow the diffusivities to vary from phase to phase (see, e.g., [31], [18]). The equations for the internal energy and solute then become

$$
\frac{\partial e}{\partial t}=\lambda \nabla^{2} T+\epsilon_{E \phi}^{2} \phi_{t} \nabla^{2} \phi+\epsilon_{E \psi}^{2} \psi_{t} \nabla^{2} \psi
$$


and

$$
\frac{\partial c}{\partial t}=\frac{D v_{m}}{R} \nabla \cdot\left[c(1-c) \nabla\left(\frac{1}{T} \frac{\partial f}{\partial c}\right)\right]
$$

The governing equations can therefore be formulated in terms of the Helmholtz free-energy density $f(T, c, \phi, \psi)$. For discussion purposes, we proceed by deriving a simple model for the free energy density that is based on ideal solutions and constant heat capacities. For example, the internal energy density for the $\alpha$-L transition for component $A$ is taken to be of the form

$$
e_{A}^{\alpha L}(T, \phi)=E_{A}+\chi_{A} T-h(\phi) L_{A}^{\alpha L}+\frac{W_{E A}^{\alpha L}}{4} g(\phi),
$$

where $E_{A}, \chi_{A}, L_{A}^{\alpha L}$, and $W_{E A}^{\alpha L}$ are constants; here $L_{A}^{\alpha L}$ is the latent heat per unit volume and $W_{E A}^{\alpha L}$ is an internal energy density barrier height for the $\alpha-L$ transition for component $A$. The heat capacity, $\chi_{A}$, is assumed to be the same for the $L, \alpha$, and $\beta$ phases of component $A$. The internal energy density for the $\beta$-L transition for component $A$ is obtained by substituting $\beta$ for $\alpha$ in the above expression. Note that both $e_{A}^{\alpha L}$ and $e_{A}^{\beta L}$ represent the same internal energy density for the bulk liquid phase, since the liquid phase is common to both the $\alpha-L$ and $\beta-\mathrm{L}$ phase transitions in this three-phase system. Analogous expressions are assumed for $e_{B}^{\alpha L}$. and $e_{B}^{\beta L}$

A corresponding entropy density for component $A$ can be written in the form

$$
s_{A}^{\alpha L}(T, \phi)=S_{A}+\chi_{A} \ln T-h(\phi) \frac{L_{A}^{\alpha L}}{T_{A}^{\alpha L}}-\frac{W_{S A}^{\alpha L}}{4} g(\phi),
$$

where $S_{A}$ is constant, and a corresponding Helmoltz free energy density for component $A$ is then

$$
\begin{aligned}
f_{A}^{\alpha L}(T, \phi)= & E_{A}-S_{A} T-\chi_{A} T \ln T+\chi_{A} T \\
& +h(\phi) \frac{L_{A}^{\alpha L}\left(T-T_{A}^{\alpha L}\right)}{T_{A}^{\alpha L}}+\frac{W_{F A}^{\alpha L}}{4} g(\phi),
\end{aligned}
$$

where $W_{F A}^{\alpha L}=W_{E A}^{\alpha L}+T W_{S A}^{\alpha L}$. The free energy density (56) agrees to within a constant with the free energy density (4) that is used in the isothermal case if the temperature is assumed to be constant. Similar expressions hold for the potentials of the $\beta-\mathrm{L}$ transition for component $A$, if $\beta$ is substituted for $\alpha$ in the above expressions. Analogous relations apply to the case of pure component $B$ as well. 
The single-component potentials can be combined using a rule-of-mixtures law, which, when we allow for the entropy of mixing gives the thermodynamic potentials for the $\alpha$-L and $\beta-\mathrm{L}$ transitions; for example, we may take

$$
\begin{gathered}
s^{\alpha L}(T, c, \phi)=c s_{B}^{\alpha L}(T, \phi)+(1-c) s_{A}^{\alpha L}(T, \phi)+\frac{R}{v_{m}} I(c), \\
e^{\alpha L}(T, c, \phi)=c e_{B}^{\alpha L}(T, \phi)+(1-c) e_{A}^{\alpha L}(T, \phi),
\end{gathered}
$$

and

$$
f^{\alpha L}(T, c, \phi)=c f_{B}^{\alpha L}(T, \phi)+(1-c) f_{A}^{\alpha L}(T, \phi)+\frac{R T}{v_{m}} I(c) ;
$$

we assume that the molar volume $v_{m}$ is constant for the system. We make similar definitions for the $\beta-\mathrm{L}$ phase transitions in the obvious manner. Finally, potential functions that are appropriate for the eutectic system are obtained by writing

$$
\begin{aligned}
& s(T, c, \phi, \psi)=h(\psi) s^{\alpha L}(T, c, \phi)+[1-h(\psi)] s^{\beta L}(T, c, \phi)-h(\phi) \frac{W_{S \psi}}{4} g(\psi), \\
& e(T, c, \phi, \psi)=h(\psi) e^{\alpha L}(T, c, \phi)+[1-h(\psi)] e^{\beta L}(T, c, \phi)+h(\phi) \frac{W_{E \psi}}{4} g(\psi),
\end{aligned}
$$

and

$$
f(T, c, \phi, \psi)=h(\psi) f^{\alpha L}(T, c, \phi)+[1-h(\psi)] f^{\beta L}(T, c, \phi)+h(\phi) \frac{W_{F \psi}}{4} g(\psi) .
$$

where $W_{F \psi}=W_{E \psi}+T W_{S \psi}$. To arrive at a tractable model we further assume that the singlecomponent internal energy densities in the liquid phase are all the same, i.e. $E_{A}=E_{B}=E_{0}$, say, and $\chi_{A}=\chi_{B}=\chi$, say. We also assume $W_{E A}^{\alpha L}=W_{E B}^{\alpha L}=W_{E A}^{\beta L}=W_{E B}^{\beta L}=W_{E \phi}$, and $S_{A}=S_{B}=S_{0}$. We then find

$$
\begin{gathered}
\frac{\partial e}{\partial t}=\chi \frac{\partial T}{\partial t}+\frac{W_{E \phi}}{4} g^{\prime}(\phi) \frac{\partial \phi}{\partial t}+\frac{W_{E \psi}}{4} h(\phi) g^{\prime}(\psi) \frac{\partial \psi}{\partial t}+\frac{W_{E \psi}}{4} g(\psi) h^{\prime}(\phi) \frac{\partial \phi}{\partial t} \\
-h^{\prime}(\phi)\left[c\left\{h(\psi) L_{B}^{\alpha L}+[1-h(\psi)] L_{B}^{\beta L}\right\}+(1-c)\left\{h(\psi) L_{A}^{\alpha L}+[1-h(\psi)] L_{A}^{\beta L}\right\}\right] \frac{\partial \phi}{\partial t} \\
-h(\phi) h^{\prime}(\psi)\left[c\left(L_{B}^{\alpha L}-L_{B}^{\beta L}\right)+(1-c)\left(L_{A}^{\alpha L}-L_{A}^{\beta L}\right)\right] \frac{\partial \psi}{\partial t} \\
-h(\phi)\left[h(\psi)\left(L_{B}^{\alpha L}-L_{A}^{\alpha L}\right)+[1-h(\psi)]\left(L_{B}^{\beta L}-L_{A}^{\beta L}\right)\right] \frac{\partial c}{\partial t}
\end{gathered}
$$


Of the seven terms on the right hand side, the first term is standard and arises from the heat capacity of the material. The second and third terms represent energy changes due to motion of the solid/liquid and solid-solid interfaces, respectively; these interfaces carry stored internal energy for non-vanishing values of $W_{E \phi}$ and $W_{E \psi}$. The fourth term contributes only near a trijunction region. The fifth and sixth terms represent the latent heat production at the solid/liquid and solid-solid interfaces, respectively. In our model the latent heat of the solid-solid transition is determined by the latent heats of the $\alpha-\mathrm{L}$ and $\beta-\mathrm{L}$ solid/liquid latent heats, and vanishes if the two latent heats for each pure component are identical. The last term accounts for the dependence of the internal energy on concentration. We note that the last term is zero in the liquid $(\phi=0)$. This is direct consequence of our making the internal energies of the pure liquid phases identical. Without this assumption the above expression would be more complicated and would allow the internal energy to vary with composition in both the the solid and liquid phases. This term vanishes if the latent heats of component $A$ and $B$ are equal for each solid/liquid transition.

The governing equations (50)-(53) provide a model of a eutectic, whose properties we investigate further below. It is worth noting that it can also be used with other thermodynamic properties of $A$ and $B$ to represent a peritectic alloy as well, although we do not pursue this here.

\subsection{Symmetric Model}

To obtain a more tractable model II for discussion purposes, we next reduce the number of parameters that appear in the governing equations by restricting our attention to the case of a eutectic alloy with a phase diagram that is symmetric about $c=1 / 2$. We also assume that $W_{F A}^{\alpha L}, W_{F B}^{\alpha L}, W_{F A}^{\beta L}$, and $W_{F B}^{\beta L}$ are all equal, and denote their common value by $W_{F \phi}$; we assume that $\epsilon_{F \phi}^{2}, \epsilon_{F \psi}^{2}, W_{F \phi}$, and $W_{F \psi}$ are constants that are independent of temperature. We further set $\epsilon_{E \phi}^{2}, \epsilon_{E \psi}^{2}, W_{E \phi}$, and $W_{E \psi}$ to zero.

For a symmetric phase diagram we require that $f(t, c, \phi, \psi)=f(T, 1-c, \phi, 1-\psi)$. From Eq. (29) we thus deduce that $f^{\alpha L}(\phi, c, T)=f^{\beta L}(\phi, 1-c, T)$. The melting temperatures for the 
pure $A \alpha-\mathrm{L}$ and pure $B \beta-\mathrm{L}$ phase transitions are equal, i.e. $T_{A}^{\alpha L}=T_{B}^{\beta L}=T_{H}$, say, and the melting temperatures for the pure $B \alpha-\mathrm{L}$ and pure $A \beta-\mathrm{L}$ transitions are equal, i.e. $T_{A}^{\beta L}=T_{B}^{\alpha L}=$ $T_{C}$, say, where we assume the labeling of the $\alpha$ and $\beta$ phases is consistent with $T_{H}>T_{C}$. It also follows that the latent heats at the hotter temperature are related by $L_{A}^{\alpha L}=L_{B}^{\beta L}=L_{H}$, and similarly at the colder temperature, we have $L_{A}^{\beta L}=L_{B}^{\alpha L}=L_{C}$. We assume the barrier heights for the Helmholtz free energy densities are equal, with $W_{F A}^{\alpha L}=W_{F B}^{\alpha L}=W_{F A}^{\beta L}=W_{F B}^{\beta L}=W_{F \phi}$.

The free energy densities in the solid phases then have the form

$$
f_{\alpha}(T, c)=\frac{R T}{v_{m}} I(c)+c L_{C} \frac{\left(T-T_{C}\right)}{T_{C}}+(1-c) L_{H} \frac{\left(T-T_{H}\right)}{T_{H}}+f_{0}(T),
$$

and

$$
f_{\beta}(T, c)=\frac{R T}{v_{m}} I(c)+(1-c) L_{C} \frac{\left(T-T_{C}\right)}{T_{C}}+c L_{H} \frac{\left(T-T_{H}\right)}{T_{H}}+f_{0}(T),
$$

where

$$
f_{0}(T)=E_{0}-S_{0} T-\chi T \ln T+\chi T .
$$

The liquid free energy density is

$$
f_{L}(T, c)=\frac{R T}{v_{m}} I(c)+f_{0}(T)
$$

In Fig. 2 we plot the dependence of the free energy curves on concentration for a fixed temperature $T$ in the range $T_{C}<T<T_{H}$.

The full Helmholtz free energy density is then given by

$$
\begin{gathered}
f(T, c, \phi, \psi)=f_{0}(T)+\frac{W_{F \phi}}{4} g(\phi)+\frac{W_{F \psi}}{4} h(\phi) g(\psi)+\frac{R T}{v_{m}} I(c)+ \\
h(\phi)\left\{L_{C} \frac{\left(T-T_{C}\right)}{T_{C}}\{h(\psi) c+[1-h(\psi)](1-c)\}\right. \\
\left.+L_{H} \frac{\left(T-T_{H}\right)}{T_{H}}\{h(\psi)(1-c)+[1-h(\psi)] c\}\right\} .
\end{gathered}
$$

In Fig. 4 we show schematically the dependence of $f$ on $\psi$ and $c$ for the solid-solid phase transition.

Note that the the solute equation (53) and the phase-field equations (50)-(51) only involve the derivatives of $f$ with respect to the variables $c, \phi$, and $\psi$, and so the term $f_{0}(T)$ in the free energy density has no effect on these governing equations. 
To further simplify the equations, it is convenient to assume that the latent heats are equal, with $L_{H}=L_{C}=L$; this simplification eliminates cross-coupling terms that would otherwise occur in the energy and solute diffusion equations, which we prefer to disregard. By varying the temperatures $T_{H}$ and $T_{C}$ and the latent heat $L$ it is possible to generate phase diagrams with a substantial variation in the degree of segregation between the liquid and solid phases. A rough estimate for the latent heats of close-packed metals is given by Richard's law, $L=1.1 R T_{M}$, where $T_{M}$ is the melting point of the transition; thus we expect that equating the latent heats is a reasonable approximation if $\left(T_{H}-T_{C}\right) / T_{C}$ is small.

\subsection{Planar Solid/Liquid Interfaces for the Pure Materials}

The gradient energy coefficients and double well barrier heights can be interpreted in terms of interface width and surface energy $[13,16]$. Consider the case of pure component $A(c=0)$. The steady-state one-dimensional phase-field $\phi(x)$ for the $\alpha$-L transition $(\psi=1)$ at the uniform temperature $T_{H}$ satisfies

$$
\epsilon_{F \phi}^{2} \phi_{x x}=f_{\phi}=\frac{W_{F \phi}}{4} g^{\prime}(\phi)
$$

with the solution

$$
\phi(x)=\frac{1}{2}\left[1-\tanh \left(\frac{x \sqrt{W_{F \phi}}}{2 \sqrt{2} \epsilon_{F \phi}}\right)\right] ;
$$

the interface width is proportional to $\epsilon_{F \phi} / \sqrt{W_{F \phi}}$. The surface energy $\sigma_{A}^{\alpha L}$ is equal to the excess Helmholtz free energy, and is given by (see, e.g., [32])

$$
\sigma_{A}^{\alpha L}=\int_{-\infty}^{\infty}\left[\frac{\epsilon_{F \phi}^{2}}{2} \phi_{x}^{2}+\frac{W_{F \phi}}{4} g(\phi)\right] d x=\frac{\epsilon_{F \phi} \sqrt{W_{F \phi}}}{6 \sqrt{2}} .
$$

The corresponding expressions for the $\beta-\mathrm{L}$ transition $(\psi=0)$ with $c=0$ at the uniform temperature $T_{C}$ have the same form, with $\sigma_{A}^{\alpha L}=\sigma_{A}^{\beta L}$. For this model, both the surface energies and the widths of the diffuse solid/liquid interfaces for the $\alpha-\mathrm{L}$ and $\beta-\mathrm{L}$ transitions for both pure materials are therefore the same. 


\subsection{Dimensionless Equations}

To proceed we non-dimensionalise the governing equations. We put:

$$
T=T_{H} \tilde{T}, \quad e=\frac{R T_{H}}{v_{m}} \tilde{e}, \quad s=\frac{R}{v_{m}} \tilde{s}, \quad f=\frac{R T_{H}}{v_{m}} \tilde{f},
$$

and set

$$
(x, y)=\mathcal{L}(\tilde{x}, \tilde{y}), \quad t=\frac{\mathcal{L}^{2}}{D} \tilde{t} .
$$

Here tilde denotes a dimensionless quantity, $\mathcal{L}$ is a characteristic length scale associated with the interfacial morphology (e.g. a lamellar spacing), and time has been scaled with the corresponding solute diffusion time. The dimensionless governing equations are

$$
\begin{gathered}
\tilde{\chi} \frac{\partial \tilde{T}}{\partial \tilde{t}}-\tilde{L} h^{\prime}(\phi) \frac{\partial \phi}{\partial \tilde{t}}=\tilde{\chi} \operatorname{Le} \tilde{\nabla}^{2} \tilde{T} \\
\frac{\partial c}{\partial \tilde{t}}=\tilde{\nabla} \cdot c(1-c) \tilde{\nabla}\left(\frac{1}{\tilde{T}} \frac{\partial \tilde{f}}{\partial c}\right) \\
\frac{\partial \phi}{\partial \tilde{t}}=\widetilde{M}_{\phi}\left(\tilde{\epsilon}_{\phi}^{2} \tilde{\nabla}^{2} \phi-\frac{\partial \tilde{f}}{\partial \phi}\right) \\
\frac{\partial \psi}{\partial \tilde{t}}=\widetilde{M}_{\psi}\left(\tilde{\epsilon}_{\psi}^{2} \tilde{\nabla}^{2} \psi-\frac{\partial \tilde{f}}{\partial \psi}\right)
\end{gathered}
$$

where

$$
\begin{gathered}
\tilde{\epsilon}_{\phi}^{2}=\frac{v_{m}}{\mathcal{L}^{2} R T_{H}} \epsilon_{F \phi}^{2}, \quad \tilde{\epsilon}_{\psi}^{2}=\frac{v_{m}}{\mathcal{L}^{2} R T_{H}} \epsilon_{F \psi}^{2}, \\
\widetilde{M}_{\phi}=\frac{R T_{H}}{v_{m}} \frac{\mathcal{L}^{2} M_{\phi}}{D}, \quad \widetilde{M}_{\psi}=\frac{R T_{H}}{v_{m}} \frac{\mathcal{L}^{2} M_{\psi}}{D}, \\
\tilde{\chi}=\frac{\chi}{\left[R / v_{m}\right]},
\end{gathered}
$$

and where the Lewis number,

$$
\mathrm{Le}=\frac{[\lambda / \chi]}{D}
$$

is the ratio of the thermal and solute diffusivities.

The dimensionless Helmholtz free energy density has the form

$$
\tilde{f}(T, c, \phi, \psi)=\frac{\widetilde{W}_{\phi}}{4} g(\phi)+\frac{\widetilde{W}_{\psi}}{4} h(\phi) g(\psi)+\tilde{T} I(c)+\tilde{f}_{0}(\tilde{T})
$$




$$
\begin{aligned}
& +h(\phi) \tilde{L}(\tau \tilde{T}-1)\{h(\psi) c+[1-h(\psi)](1-c)\} \\
& +h(\phi) \tilde{L}(\tilde{T}-1)\{h(\psi)(1-c)+[1-h(\psi)] c\}
\end{aligned}
$$

where $\tau=T_{H} / T_{C}$ and

$$
\widetilde{W}_{\phi}=\frac{W_{F \phi}}{\left[R T_{H} / v_{m}\right]}, \quad \widetilde{W}_{\psi}=\frac{W_{F \psi}}{\left[R T_{H} / v_{m}\right]}, \quad \tilde{L}=\frac{L}{\left[R T_{H} / v_{m}\right]} .
$$

The dimensionless function $\tilde{f}_{0}=v_{m} f_{0} /\left[R T_{H}\right]$ again plays no role in the subsequent analysis. An expression for the diffusion potential is then given by

$$
\tilde{\mu}=\tilde{f}_{c}=\tilde{T} I^{\prime}(c)+h(\phi) \tilde{L}(\tau-1) \tilde{T}[2 h(\psi)-1]
$$

so that the expression $\tilde{\mu} / \tilde{T}$ is independent of temperature in this model.

The dimensionless internal energy density has the form

$$
\tilde{e}(T, c, \phi, \psi)=\tilde{E}_{0}+\tilde{\chi} \tilde{T}-\tilde{L} h(\phi) .
$$

where $\tilde{E}_{0}=v_{m} E_{0} /\left[R T_{H}\right]$. The dimensionless solid/liquid surface energy is defined by $\tilde{\sigma}_{L}=$ $\sigma /\left(\mathcal{L}\left[R T_{H} / v_{m}\right]\right)$.

\section{A Trijunction in the Sharp Interface Limit, $\tilde{\epsilon}_{\phi} \rightarrow 0, \tilde{\epsilon}_{\psi} \rightarrow 0$.}

We next consider a trijunction consisting of the confluence of two solid/liquid interfaces (between the liquid and the $\alpha$ solid and the liquid and the $\beta$ solid) and a solid/solid interface (between the $\alpha$ and $\beta$ solid phases). We analyse this situation in the sharp interface limit where the diffuse interfaces may be approximated by curves in the plane. In this limit, the gradient energy coefficients are assumed small, and the barrier heights large, with the scales chosen in such a way that the surface energies remain finite as the interface thickness tends to zero (see, e.g., [17]). The barrier heights of the free energy density are then written

$$
\widetilde{W}_{\phi}=\frac{\widehat{W}_{\phi}}{\tilde{\epsilon}_{\phi}^{2}}, \quad \widetilde{W}_{\psi}=\frac{\widehat{W}_{\psi}}{\tilde{\epsilon}_{\psi}^{2}} ;
$$

we note that $\widehat{W}_{F \phi}=72 \tilde{\sigma}_{L}^{2}$. 
The dimensionless Helmholtz free energy density may then be expressed as

$$
\tilde{f}=\tilde{f}^{(-2)}(\phi, \psi)+\tilde{f}^{(0)}(\tilde{T}, c, \phi, \psi),
$$

where

$$
\tilde{f}^{(-2)}(\phi, \psi)=\frac{\widehat{W}_{\phi}}{4 \tilde{\epsilon}_{\phi}^{2}} g(\phi)+\frac{\widehat{W}_{\psi}}{4 \tilde{\epsilon}_{\psi}^{2}} h(\phi) g(\psi),
$$

and

$$
\begin{gathered}
\tilde{f}^{(0)}(\tilde{T}, c, \phi, \psi)=\tilde{T} I(c)+\tilde{f}_{0}(\tilde{T}) \\
+h(\phi) \tilde{L}(\tau \tilde{T}-1)\{h(\psi) c+[1-h(\psi)](1-c)\} \\
+h(\phi) \tilde{L}(\tilde{T}-1)\{h(\psi)(1-c)+[1-h(\psi)] c\} .
\end{gathered}
$$

Further, we consider the distinguished limit in which the mobilities of both the solid/solid and solid/liquid interfaces are very large, which is typical of metallic systems where local equilibrium is a good approximation. We set

$$
\widetilde{M}_{\phi}=\frac{\widetilde{m}_{\phi}}{\tilde{\epsilon}_{\phi}^{2}}, \quad \widetilde{M}_{\psi}=\frac{\widetilde{m}_{\psi}}{\tilde{\epsilon}_{\psi}^{2}} .
$$

We will also assume that

$$
\tilde{\epsilon}_{\psi}=\Lambda \tilde{\epsilon}_{\phi}
$$

where $\Lambda$ is an order one quantity. In this distinguished limit the interfacial layers are of thickness $\mathcal{O}\left(\tilde{\epsilon}_{\phi}^{2}\right)$ and so we expect the dimensions of the trijunction region where they meet to be also characterized by the same length scale. In the next subsection we consider the outer problem representing the solution outside the interfacial layers and away from the trijunction region. We then go on to consider, in subsequent subsections, the solution in the solid/liquid and solid/solid interfacial layers and finally the trijunction region.

\subsection{The Outer Solution}

The outer solution comprises three different regions; the liquid region, here denoted by $\mathrm{L}$, and the two regions where the $\alpha$ and $\beta$ solid phases exist, denoted by $\alpha$ and $\beta$. This situation is illustrated in Fig. 6. 
In both solid regions $\phi \equiv 1$ and, in the $\alpha$ and $\beta$ solid regions, $\psi \equiv 1$ and 0 respectively. The temperature and solute concentration satisfy the conventional diffusion equations.

In the liquid region again the temperature and solute concentration satisfy the diffusion equation and $\phi=0$. However, the situation for $\psi$ is more complicated. Whereas in $\alpha$ and $\beta$ solid regions the leading-order bulk values for $\phi$ and $\psi$ are determined by the minima in the free energy function, in the liquid region the free energy is constructed so that there is no dependence on $\psi$ to leading order. Consequently, a regular perturbation expansion for $\psi$ in terms of $\epsilon_{\psi}^{2}$ shows that $\psi$ satisfies Laplace's equation to leading order. As we show below, at leading order $\psi$ is continuous across the solid/liquid interfaces, and hence $\psi$ varies smoothly throughout the liquid region with values lying between zero and one. This behavior is observed clearly in numerical calculations which are described below, see Fig. 7. We emphasize, however, that the specific form assumed by $\psi$ in the bulk liquid region have no effect on the state of the system since the free energy in the bulk liquid is independent of $\psi$.

\subsection{The Solid/Liquid Interfaces}

We next consider an inner solution which describes the $\alpha-I$ interface at a distance far from the trijunction region; the results for the $\beta$-L interface for $\psi=0$ are analogous.

Following [31], we introduce a local orthogonal coordinate system $(r, s)$ moving with the interfacial layer, where $r$ measures distance across the interfacial layer and $r(x, y)=0$ represents the level set $\phi^{(0)}=1 / 2$, with positive values of $r$ corresponding to the liquid phase $(\phi=0)$. We scale $r$ to the thickness of the interfacial layer by writing $r=\tilde{\epsilon}_{\phi}^{2} \rho$ and expand the field variables in the layer as a perturbation series in $\tilde{\epsilon}_{\phi}^{2}$,

$$
\begin{aligned}
& \widehat{\phi}=\widehat{\phi}^{(0)}+\widehat{\phi}^{(2)} \tilde{\epsilon}_{\phi}^{2}+O\left(\tilde{\epsilon}_{\phi}^{4}\right), \\
& \widehat{\psi}=\widehat{\psi}^{(0)}+\widehat{\psi}^{(2)} \tilde{\epsilon}_{\phi}^{2}+O\left(\tilde{\epsilon}_{\phi}^{4}\right), \\
& \widehat{c}=\widehat{c}^{(0)}+\widehat{c}^{(2)} \tilde{\epsilon}_{\phi}^{2}+O\left(\tilde{\epsilon}_{\phi}^{4}\right), \\
& \widehat{T}=\widehat{T}^{(0)}+\widehat{T}^{(2)} \tilde{\epsilon}_{\phi}^{2}+O\left(\tilde{\epsilon}_{\phi}^{4}\right),
\end{aligned}
$$


where the hats denote the dependent variables in the interfacial layer. It is convenient to write a formal expansion for the chemical potential as well, with

$$
\widehat{\mu}=\widehat{\mu}^{(0)}+\widehat{\mu}^{(2)} \tilde{\epsilon}_{\phi}^{2}+O\left(\tilde{\epsilon}_{\phi}^{4}\right) .
$$

We find that the leading order temperature $\widehat{T}^{(0)}$ in the layer satisfies

$$
\widehat{T}_{\rho p}^{(0)}=0
$$

and matching with the outer solution for large $\rho$ shows that the inner solution is constant and the outer solution is continuous across the interface.

The leading order phase field $\widehat{\psi}^{(0)}$ satisfies

$$
\Lambda^{2} \widehat{\psi}_{\rho \rho}^{(0)}-\frac{\widehat{W}_{\psi}}{4 \Lambda^{2}} h\left(\hat{\phi}^{(0)}\right) g^{\prime}\left(\hat{\psi}^{(0)}\right)=0
$$

The solutions to this equation that are of interest for the solid/liquid interface are the constant solutions with $\hat{\psi}^{(0)}=0$ or 1 . Matching with the outer solution in the $\alpha$-region requires that $\widehat{\psi}^{(0)}=1$.

The leading order phase field $\widehat{\phi}^{(0)}$ is given by

$$
\widehat{\phi}^{(0)}=\frac{1}{2}\left[1-\tanh \left(3 \tilde{\sigma}_{L} \rho\right)\right] .
$$

The leading order diffusion potential, $\widehat{\mu}^{(0)}$, is constant across the interfacial layer,

$$
\widehat{\mu}^{(0)}\left(\widehat{T}^{(0)}, \widehat{c}^{(0)}, \widehat{\phi}^{(0)}, 1\right)=\widehat{\mu}_{0}
$$

the value of $\widehat{\mu}_{0}$ is undetermined at this stage. It is convenient to define

$$
F(T, c, \phi)=\tilde{f}^{(0)}(T, c, \phi, 1)-\widehat{\mu}_{0} c,
$$

so that Eq. (99) can be written as $F_{c}\left(\widehat{T}^{(0)}, \hat{c}^{(0)}, \widehat{\phi}^{(0)}\right)=0$.

At next order in the interfacial layer, $O\left(\tilde{\epsilon}_{\phi}^{2}\right)$, we find that

$$
\widehat{\phi}_{\rho \rho}^{(2)}-\frac{\widetilde{W}_{\phi}}{4} g^{\prime \prime}\left(\widehat{\phi}^{(0)}\right) \widehat{\phi}^{(2)}=F_{\phi}\left(\widehat{T}^{(0)}, \widehat{c}^{(0)}, \widehat{\phi}^{(0)}\right)-\left(\frac{\tilde{V}_{n}}{\widetilde{m}_{\phi}}+\tilde{K}\right) \widehat{\phi}_{\rho}^{(0)}
$$




$$
\begin{gathered}
{\left[\frac{\widehat{c}^{(0)}\left(1-\widehat{c}^{(0)}\right)}{\widehat{T}(0)} \hat{\mu}_{\rho}^{(2)}\right]_{\rho}+V_{n} \widehat{c}_{\rho}^{(0)}=0,} \\
\chi L e \widehat{T}_{\rho \rho}^{(2)}=\tilde{L} \tilde{V}_{n} h^{\prime}\left(\widehat{\phi}^{(0)}\right) \widehat{\phi}_{\rho}^{(0)}
\end{gathered}
$$

where $\tilde{V}_{n}$ and $\tilde{K}$ are the dimensionless normal velocity and local curvature of the interface, respectively. The normal velocity is positive for motion of the interface into the liquid (freezing) and the curvature positive for solid projections into the liquid. Eq. (101) gives a solvability condition, which after matching with the solution in the bulk phases yields

$$
\left.F\left(\phi^{(0)}, \tilde{T}^{(0)}, c^{(0)}\right)\right|_{S} ^{L}=\tilde{\sigma}_{L}\left(\frac{\tilde{V}_{n}}{{\widetilde{m_{\phi}}}}+\tilde{K}\right),
$$

where $L$ and $S$ denote evaluation of the quantity at the liquid and solid side of the sharp interface. Further, Eq. (102) and Eq. (103) may be integrated once, which on application of the matching conditions gives that

$$
\left.\frac{\partial c^{(0)}}{\partial n}\right|_{S} ^{L}+\left.\tilde{V}_{n} c^{(0)}\right|_{S} ^{L}=0
$$

and

$$
\left.\tilde{\chi} L e \frac{\partial \tilde{T}^{(0)}}{\partial n}\right|_{S} ^{L}=-\tilde{L} \tilde{V}_{n},
$$

which simply represent conservation of solute and internal energy across the interface. Eq. (99) evaluated at $\rho= \pm \infty$ gives

$$
\tilde{\mu}_{0}=\tilde{f}_{c}^{(0)}\left(\tilde{T}^{(0)}, c_{S}, 1,1\right)=\tilde{f}_{c}^{(0)}\left(\tilde{T}^{(0)}, c_{L}, 0,1\right)
$$

Eq. (104) and Eq. (107) together provide the relationship between the leading order interfacial concentrations in the solid and liquid, here denoted by by $c_{S}$ and $c_{L}$ respectively, in the sharp interface limit. In fact they show that the interfacial concentrations are related by a parallel tangent construction. This is a consequence of the leading order diffusion potential being continuous across the interface. For the particular case of a stationary flat interface, $\tilde{V}_{n}=\tilde{K}=0$, it reduces to a common tangent construction, which determines the leading order equilibrium interfacial concentrations, $c_{S}$ and $c_{L}$, in the solid and liquid respectively in terms of the interfacial temperature, $\tilde{T}_{I}$. From this we are able to determine the liquidus and solidus curves for the $\alpha-\mathrm{L}$ and $\beta-\mathrm{L}$ phase transitions, as shown schematically in Fig. 5. We see that the liquidus curves and 
solidus curves of the $\alpha-\mathrm{L}$ and $\beta-\mathrm{L}$ systems are both given by simple lens shapes. The liquidus curves intersect at the eutectic temperature and eutectic composition. This is the special liquid composition that can be at equilibrium with both $\alpha$ and $\beta$. Below this temperature, the liquidus and solidus curves become metastable.

In Appendix A we use this parallel tangent construction to obtain expressions for the slope of the solidus curves and liquidus curves on the phase diagram (at $V=\tilde{K}=0$ ), as well as show that for dilute alloys it results in the following interfacial temperature condition, expressed in dimensional form, as

$$
T_{I}=T_{H}+\left[\frac{\left(k^{\alpha L}-1\right) R T_{H}^{2}}{v_{m} L_{A}}\right] c_{L}-\frac{\sigma^{\alpha L} T_{H}}{L_{A}} K-\frac{V_{n}}{\mu^{\alpha L}} .
$$

This second term on the right is the classical form for the liquidus slope for an ideal solution model of a dilute alloy. The second and third terms represent the classical forms of the GibbsThomson effect and interfacial kinetics.

We therefore recover, at leading order independent of $\tilde{\epsilon}_{\phi}$, the classical form of the interface boundary conditions in the sharp interface limit, in which both internal energy and solute are conserved across the interface and the Gibbs-Thomson effect and interface kinetics are included correctly.

\subsection{The Solid/Solid Interface}

We next consider the solid/solid interface for which $\phi=1$. For a planar stationary interface, the equilibrium concentrations $c^{\alpha}$ and $c^{\beta}$ in each phase are related by a common tangent construction. Because the free energy is invariant under $c \rightarrow(1-c)$ and $\psi \rightarrow(1-\psi)$, it follows that $c^{\alpha}$ is given by the minima of the free energy with respect to $c$ with $\psi=1$, with a similar result for $c^{\beta}$. This gives that the $\alpha$ solid concentration at the interface is

$$
c^{\alpha}=\frac{1}{1+\exp [\tilde{L}(\tau-1)]}
$$

and $c^{\beta}=1-c^{\alpha}$. We note that in this model the equilibrium concentrations are independent of the temperature, and so the solvus curves on the phase diagram are vertical lines; this is a result of the high degree of symmetry assumed in the model. 
The sharp interface analysis for the solid/solid phase transition between the $\alpha$ and $\beta$ phases, in the limit $\tilde{\epsilon}_{\psi} \rightarrow 0$, is, by design, similar to that given above for the solid/liquid interface and yields both conservation of solute and internal energy, as well a parallel tangent construction relating the interfacial concentrations to the normal velocity and local curvature of the interface. To leading order, the variation of the phase field $\psi$ through the interface is given by a hyperbolic tangent profile as in Eq. (98); the dimensionless solid/solid surface tension $\tilde{\sigma}_{S}$ satisfies $\widehat{W}_{\psi}=$ $72 \tilde{\sigma}_{S}^{2}$.

\subsection{The Trijunction Region}

We next analyse a stationary trijunction between the two solid phases and the liquid. Our analysis is based on related work of Bronsard and Reitich [33], who included a heuristic derivation, in the sharp-interface limit, of the appropriate form of Young's law at a trijunction for a model with three order parameters. Our work generalizes their treatment in that our model contains two order parameters, $\phi$ and $\psi$, together with the temperature and solute field. In their work, the outer solutions consisted of uniform states, whereas in our work the $\psi$ order parameter varies throughout the bulk liquid phase. We provide a similar formal analysis for the equilibrium angles formed at the triple junction, with the appropriate modifications necessary to account for the variation of the outer solution in the bulk phases.

Specifically we discuss a eutectic trijunction with a symmetrical geometry that also forms a basis for the numerical computations in the following section. To avoid further technical complications associated with the behavior of the outer solution, in this section we assume that the trijunction is isothermal and stationary. We therefore consider a trijunction at the eutectic temperature with straight solid/liquid interfaces that are arranged symmetrically on either side of the solid/solid interface, which is taken to lie in the plane of symmetry. The compositions in each phase are consequently uniform, and because of the assumed symmetry of the free energy they are related by $c^{\alpha}=1-c^{\beta}$ and $c^{L}=1 / 2$ through the common tangent construction.

We introduce a control triangle, denoted by $T$, about the trijunction, such that the interfaces of the trijunction form the perpendicular bisectors of each side of the triangle, see Fig. 6 . We 
position each face of the triangle to be equidistant from the centre of the trijunction (where $\phi=\psi=1 / 2)$ at a distance, $d$. We shall consider the sharp interface limit, by considering the double limit, $\tilde{\epsilon}_{\phi}, \tilde{\epsilon}_{\psi} \rightarrow 0$, with $\tilde{\epsilon}_{\psi}=\Lambda \tilde{\epsilon}_{\phi}$, followed by $d \rightarrow 0$, i.e. we first shrink the interface thickness within the bounding triangle $T$, and then shrink $T$ about the trijunction.

Under these assumptions, the governing equation for the solute field can be integrated to give

$$
\tilde{f}_{c}=0
$$

where a constant of integration, which represents the slope of the common tangent, is zero for this symmetric case.

For the phase-field variables, it is convenient to introduce the notation $\tilde{\mathbf{u}}=(\tilde{\phi}, \tilde{\psi})^{T}$, The equations for the phase field variables then can be written in the form

$$
D \tilde{\nabla}^{2} \tilde{\mathbf{u}}-\tilde{\mathrm{f}}_{\tilde{u}}(\tilde{\mathbf{u}})=0
$$

where $\tilde{\mathrm{f}}_{\tilde{u}}(\tilde{\mathbf{u}})=\left(\tilde{f}_{\phi}, \tilde{f}_{\psi}\right)^{T} . D$ is a diagonal matrix having the form

$$
D=\left(\begin{array}{cc}
\tilde{\epsilon}_{\phi}^{2} & 0 \\
0 & \Lambda^{2} \tilde{\epsilon}_{\phi}^{2}
\end{array}\right) \text {. }
$$

We now integrate Eq. (111) over the triangle, $T$. To do so we first introduce some definitions and notation, as illustrated in Fig. 6. At each intersection point of an interface with a side of the triangle, each interface is inclined at an angle $\gamma_{i}$ to the horizontal and a local Cartesian coordinate $\operatorname{system}\left(\tilde{\zeta}_{i}, \tilde{\zeta}_{i}\right)$ is associated with each side of the triangle. The $\tilde{\zeta}_{i}$ coordinate direction is tangent to its associated interface and the corresponding $\tilde{\xi}_{i}$ coordinate is normal to the interface at the intersection point. The $\tilde{\zeta}_{i}$ all increase towards the centre of $T$. The three local coordinate systems are related by rotations, with

$$
\left(\begin{array}{c}
\tilde{\xi}_{i} \\
\tilde{\zeta}_{i}
\end{array}\right)=\left(\begin{array}{cc}
-\sin \left(\gamma_{i}\right) & \cos \left(\gamma_{i}\right) \\
-\cos \left(\gamma_{i}\right) & -\sin \left(\gamma_{i}\right)
\end{array}\right)\left(\begin{array}{c}
\tilde{\xi}_{1} \\
\tilde{\zeta}_{1}
\end{array}\right),
$$

for $i=2,3$; i.e., rotations by $\pi / 2+\gamma_{i}$. It is convenient to define an inner product $\mathbf{u} \star \mathbf{v}=(A \mathbf{u}) \cdot \mathbf{v}$, i.e. an inner product under a non-Euclidean metric; here dot is the usual Euclidean inner 
product. Taking the Euclidean inner product of the governing equations (111) with $\mathbf{u}_{\tilde{\xi}_{1}}^{(0)}$ and integrating over the triangle $T$ gives

$$
\int_{T} \tilde{\mathbf{u}}_{\tilde{\xi}_{1}} \star \tilde{\nabla}_{1}^{2} \tilde{\mathbf{u}} d \tilde{a}_{1}-\int_{T} \tilde{\mathbf{u}}_{\tilde{\xi}_{1}} \cdot \tilde{\mathbf{f}}_{\tilde{u}}(\tilde{\mathbf{u}}) d \tilde{a}_{1}=0
$$

where $d \tilde{a}_{i}=d \tilde{\xi}_{i} d \tilde{\zeta}_{i}, i=1,2,3$ is the differential area element. We now note the following identity, which is easily proved:

$$
\left[\tilde{\mathbf{u}}_{\tilde{\xi}_{1}} \star \tilde{\mathbf{u}}_{\tilde{\zeta}_{1}}\right]_{\tilde{\xi}_{1}}=\tilde{\mathbf{u}}_{\tilde{\xi}_{1}} \star \widehat{\nabla}^{2} \tilde{\mathbf{u}}+\frac{1}{2}\left(\left\|\tilde{\mathbf{u}}_{\tilde{\zeta}_{1}}\right\|_{\tilde{\xi}_{1}}^{2}-\left\|\tilde{\mathbf{u}}_{\tilde{\xi}_{1}}\right\|_{\tilde{\xi}_{1}}^{2}\right)
$$

where $\|\tilde{\mathbf{u}}\|^{2}=\tilde{\mathbf{u}} \star \tilde{\mathbf{u}}$. Thus the above expression may be written as

$$
\int_{T}\left\{\frac{\partial}{\partial \tilde{\zeta}_{1}}\left[\tilde{\mathbf{u}}_{\tilde{\xi}_{1}} \star \tilde{\mathbf{u}}_{\tilde{\zeta}_{1}}\right]+\frac{\partial}{\partial \tilde{\xi}_{1}}\left[\frac{1}{2}\left(\left\|\tilde{\mathbf{u}}_{\tilde{\xi}_{1}}\right\|^{2}-\left\|\tilde{\mathbf{u}}_{\tilde{\zeta}_{1}}\right\|^{2}\right)-\tilde{f}\right]\right\} d \tilde{a}_{1}=0
$$

where we have used the fact that $\tilde{f}_{c}=0$ in order to express the integrand in divergence form. We now employ the divergence theorem to obtain that

$$
\sum_{i=1}^{i=3} \int_{\partial T_{i}}\left\{\left[\tilde{\mathbf{u}}_{\tilde{\xi}_{1}} \star \tilde{\mathbf{u}}_{\tilde{\zeta}_{1}}\right] n_{2}^{i}+\left[\frac{1}{2}\left(\left\|\tilde{\mathbf{u}}_{\tilde{\xi}_{1}}\right\|^{2}-\left\|\tilde{\mathbf{u}}_{\tilde{\zeta}_{1}}\right\|^{2}\right)-\tilde{f}\right] n_{1}^{i}\right\} d \tilde{\xi}_{i}=0
$$

where $\mathbf{n}=\left(n_{1}^{i}, n_{2}^{i}\right)=\left(\cos \left(\gamma_{i}\right), \sin \left(\gamma_{i}\right)\right)$ is the outward unit normal vector to the side $i$ of the triangle $T$, denoted by $\partial T_{i}$.

There are two types of leading order contributions to the above integral: one is from the behavior of the inner solution at the interfacial layers [33], and the other is from the variation of the outer solution along the sides of the triangle. The former contribution is of order unity independent of the length $d$ of the triangle sides, and the latter is bounded by product of the maximum of the integrand and the length of the triangle sides, and does not contribute to the final result in the limit that $d \rightarrow 0$. We therefore discuss the considerations appropriate to the contribution from the interfacials layers, with error terms that are formally of order $d$.

In the interfacial layers away from the center of the triangle, to leading order the solutions vary only in the direction parallel to the triangle sides, and we may write

$$
\frac{\partial}{\partial \tilde{\xi}_{1}}=-\sin \gamma_{i} \frac{\partial}{\partial \tilde{\xi}_{i}}
$$




$$
\frac{\partial}{\partial \tilde{\zeta}_{1}}=\cos \gamma_{i} \frac{\partial}{\partial \tilde{\xi}_{i}},
$$

If we denote the contribution from the outer solution as $O(d)$, we obtain the approximation

$$
\sum_{i=1}^{i=3} \cos \left(\gamma_{i}\right) \int_{\partial T_{i}}\left\{\frac{1}{2}\left\|\mathbf{u}_{\tilde{\xi}_{i}}\right\|^{2}+\widehat{f}(\widehat{\mathbf{u}})\right\} d \tilde{\xi}_{i}=O(d) .
$$

Noting that the surface tension of the $i$ ' th interface, $\tilde{\sigma}_{i}$, is the Helmholtz free energy excess which is given by

$$
\tilde{\sigma}_{i}=\int_{-\infty}^{+\infty}\left\{\frac{1}{2}\left\|\mathbf{u}_{\tilde{\xi}_{i}}\right\|^{2}+\hat{f}\right\} d \tilde{\xi}_{i},
$$

then Eq. (120) may be rearranged to give

$$
\boldsymbol{\Sigma}_{1}=O(d)
$$

where $\boldsymbol{\Sigma}_{i}$ is the component (at leading order in $\tilde{\epsilon}_{\phi}^{2}$ ), in the direction perpendicular to the $i^{\prime}$ th interface, of the net force, $\boldsymbol{\Sigma}$, acting on the trijunction due to the surface tensions of the three interfaces. Thus $\Sigma=\sum_{i=1}^{i=3} \tilde{\sigma}_{i} \mathbf{e}_{i}$, where $\mathbf{e}_{i}$ are the three unit vectors parallel to the interfaces.

This procedure may be repeated by taking the inner product with $\mathbf{u}_{\xi_{2}}$ giving the analogous result $\Sigma_{2}=O(d)$, from which we deduce that the net force due to the surface tensions, acting on the trijunction at leading order, is of order d, i.e. $\Sigma=O(d)$. Having taken the limit of vanishing $\tilde{\epsilon}_{\phi}$, we may then consider the additional limit that $d \rightarrow 0$, in which case we obtain Young's law in the form

$$
\Sigma=0 .
$$

We note that if we instead consider interfaces with a small but finite thickness, it is inappropriate to let the triangle area tend to zero, and the above force balance is modified by the additional $O(d)$ contributions, which are suggestive of the effects of a line energy of the trijunction.

\section{Computations}

We next discuss numerical solutions of the steady governing equations for phase-field model II with the aim of describing a stationary trijunction located in a linear temperature gradient. In particular we consider the special case of the symmetric model discussed in section 3.1 in which 
all the latents heats are equal. We prescribe the temperature to be $\tilde{T}=\tilde{T}_{E}+\tilde{G} \tilde{y}$, where $\tilde{G}$ is the dimensionless temperature gradient, and $\widetilde{T}_{E}$ is the dimensionless eutectic temperature. As noted in section 4.3 the solvus curves are vertical and hence the eutectic concentrations in the solid $\alpha$ and solid $\beta$ phases are given in Eq. (109).

\subsection{Sharp Interface Solution}

We first consider the solution to be expected in the sharp interface limit $\tilde{\epsilon}_{\phi} \rightarrow 0, \tilde{\epsilon}_{\psi} \rightarrow 0$. In the case of a eutectic phase diagram that is symmetric with respect to the composition $(c \rightarrow(1-c)$ ) with both the $\alpha-\mathrm{L}$ and $\beta$-L surface energies equal, a trijunction exists that consists of a planar $\alpha-\beta$ interface which has reflection symmetry about the vertical axis. The two solid/liquid interfaces subtend the same angle to the horizontal $\theta$, given by $2 \tilde{\sigma}_{L} \sin (\theta)=\tilde{\sigma}_{S}$. For this reason, we henceforth only consider the right-hand side of the trijunction, with the solid/solid interface located about $x=0$. The steady-state solute equation Eq. (75) with the appropriate no-flux boundary conditions gives that the diffusion potential $f_{c}$ is constant in each phase, and by continuity of the diffusion potential across the three interfaces it must have the same value everywhere. Moreover, as noted in section 4.4 the diffusion potential at the solid/solid interface is zero and it is therefore zero throughout the entire domain. As a consequence the concentration in each phase is constant; in the liquid it is $1 / 2$ and in the two solid phases it has the appropriate eutectic compositions, $c_{S}^{\alpha L}$ and $c_{S}^{\beta L}$, given by Eq. (109). It follows from the parallel tangent construction, given by Eq. (104) and Eq. (107), which emerges from the sharp interface limit, that the position of the $\beta-\mathrm{L}$ interface, denoted by $y=h(x)$, satisfies

$$
h=\frac{B h^{\prime \prime}}{\left[1+\left(h^{\prime}\right)^{2}\right]^{3 / 2}}, 0<x<1,
$$

with the boundary conditions $h^{\prime}(0)=\tan (\theta)$ and $h^{\prime}(1)=0$, where

$$
B=\frac{\tilde{\sigma}_{L}}{\tilde{G}\left\{\tilde{L}\left[c_{S}^{\beta L}(\tau-1)+1\right]+I\left(c_{S}^{\beta L}\right)-I\left(c_{L}^{\beta L}\right)\right\}} .
$$

This ordinary differential equation is easily solved numerically, and the solutions can be used to compare with numerical solutions of the phase-field equations, as discussed in the next subsection. 


\subsection{Numerical Method and Results}

In the light of the symmetry of the trijunction discussed above and the fact that the diffusion potential is everywhere zero, we solved

$$
\begin{gathered}
\tilde{\epsilon}_{\phi}^{2} \nabla^{2} \phi-\tilde{f}_{\phi}(\phi, \psi, c, \tilde{T})=0, \\
\tilde{\epsilon}_{\psi}^{2} \nabla^{2} \phi-\tilde{f}_{\psi}(\phi, \psi, c, \tilde{T})
\end{gathered}
$$

with

$$
\tilde{f}_{c}(\phi, \psi, c, \tilde{T})=0
$$

on the domain $0<x<1$ and $-1 / 2<y<1 / 2$. We imposed the boundary conditions

$$
\frac{\partial \phi}{\partial n}=\frac{\partial \psi}{\partial n}=\frac{\partial c}{\partial n}=0
$$

on the boundaries along $x=1, y= \pm 1 / 2$ (the latter representing no solute flux across the boundary of the domain), and

$$
c=\psi=\frac{1}{2} \text { and } \frac{\partial \phi}{\partial n}=0,
$$

representing the symmetry boundary conditions along $x=0$. Eq. (128) can be rearranged to give $c=\Omega(\phi, \psi)$, where

$$
\Omega(\phi, \psi)=\frac{1}{1+\exp [\tilde{L}(\tau-1) h(\phi)(2 h(\psi)-1)]} .
$$

With $c$ so determined we solved Eq. (126) and Eq. (127) for $\phi$ and $\psi$ with their associated boundary conditions.

We used a standard five point finite difference approximation of the Laplacian on a uniform grid to discretize the governing equations (126) and (127). The resulting system of nonlinear algebraic equations was solved using Newton iteration. The sparse symmetric linear system that arises was solved by a preconditioned iterative technique using the NSPCG [34] software package. It was found that a first order Neumann polynomial preconditioner allied to the biconjugate gradient squared accelerator provided the most computationally efficient combination. Continuation was used, typically by varying $\tilde{\sigma}_{S}$. All the computations shown here were done on a $128 \times 128$ mesh. 


\begin{tabular}{|l|l|}
\hline Parameter & Value \\
\hline$\tilde{\epsilon}_{\phi}$ & 0.08 \\
$\tilde{\epsilon}_{\psi}$ & 0.08 \\
$\tilde{L}_{\tilde{\sigma}}$ & 0.1 \\
$\tilde{\sigma}_{L}$ & 0.2 \\
$\tilde{G}$ & 0.187 \\
$\tau$ & 10.0 \\
\hline
\end{tabular}

Table 1: The values of the dimensionless parameters used in the computations

The results shown in Fig. 7 and Fig. 8 have been reflected about $x=0$ to show both sides of the trijunction. In Fig. 7 we show a well-developed trijunction; the values of the dimensionless parameters used in the numerical computations are given in Table 1. This figure depicts the $\phi, \psi$ and $c$ fields. In (a) we show the solute field. The contours are at 5\%,50\% and $95 \%$ of the range and show that the concentration variation is confined to the interfacial regions as expected from the sharp interface solution. In (b) we show contours of the $\psi$ phase-field. The $\alpha-\beta$ interface is given is clearly apparent, across which $\psi$ varies rapidly in the solid. In the liquid the $\psi$ contours fan out. The variation of $\psi$ in the liquid, where $\phi$ is zero, does not affect the value of the Helmholtz free-energy functional there. In (c) we show the $\phi$ phase-field; three contours are shown, corresponding to $\phi=0.05,0.5$ and 0.95 . We see that the the solid/liquid interface curves down to meet the trijunction as expected. The diffuse trijunction region where both $\phi$ and $\psi$ vary is very much confined to the intersection of the solid/liquid and solid/solid interfacial regions as expected. In (c) the solid circles show the position of the interface given by the sharp interface solution, found by solving Eq. (124). There is clearly excellent agreement between the phase-field and sharp interface solutions. In Fig. 8 we show the $\phi$ phase fields for three different values of the solid/solid surface energy. The surface energy successively decreased from (a) to (c). The parameter values were the same as those used previously, given in Table 1, except that $\tilde{\epsilon}_{\phi}=\tilde{\epsilon}_{\psi}=0.1, \tilde{L}=1$ and $\tilde{G}=1$. This larger value of the latent heat results in a greater degree of solute segregation across the interface, and the agreement with the sharp interface solution, again represented by the solid circles, is not as good in this case. The computed phase- 
field interfacial layer is uniformly displaced from the sharp interface solution. Despite this we observe the correct qualitative behaviour, with the interface becoming more curved and being displaced further behind the eutectic isotherm $(y=0)$ as $\tilde{\sigma}_{S}$ is increased.

\section{Conclusions}

In this paper we have considered two phase-field models of a eutectic alloy. The first, based on a regular solution model with a chemical miscibility gap suffers from the deficiency that, in the sharp interface limit the solid/solid surface energy is zero. We went on to develop an alternate phase-field model which uses two order parameters to represent the different phases and have provided a thermodynamically consistent derivation of this phase-field model which guarantees that the local entropy production is positive. A sharp interface asymptotic analysis of the liquid/solid phase transition result in a free-boundary problem in which both surface energy and interface kinetics are present. Finally, we have considered a sharp interface asymptotic analysis of a stationary, isothermal, trijunction from which we demonstrated that the interfacial surface tensions are in mechanical equilibrium. This is compared favourably with numerical solutions of our model appropriate to a trijunction.

\section{Acknowledgments}

The authors are grateful for discussions with R. J. Braun, S. R. Coriell, Martin Grant, A. Karma, and B. T. Murray. This work was performed with support from the Applied and Computational Mathematics Program of DARPA and the Microgravity Science and Applications Program of NASA. 


\section{A. The Parallel Tangent Construction}

From the common tangent construction Eq. (104) and Eq. (107) it is a straight forward matter to show that the slope of the liquidus and solidus in for $\alpha-\mathrm{L}$ phase transitions are given as

$$
\begin{aligned}
& \widetilde{m}_{S}\left(\tilde{T}_{I}\right)=\frac{d \tilde{T}_{I}}{d c_{S}}=\tilde{f}_{\mathrm{cc}}\left(c_{S}\right) /\left[\frac{\tilde{f}_{\widetilde{T}_{I}}\left(c_{S}\right)-\tilde{f}_{\widetilde{T}_{I}}\left(c_{L}\right)}{c_{S}-c_{L}}-\tilde{f}_{c \widetilde{T}_{I}}\left(c_{S}\right)\right], \\
& \widetilde{m}_{L}\left(\tilde{T}_{I}\right)=\frac{d \widetilde{T}_{I}}{d c_{L}}=\tilde{f}_{c c}\left(c_{L}\right) /\left[\frac{\tilde{f}_{\widetilde{T}_{I}}\left(c_{S}\right)-\tilde{f}_{\widetilde{T}_{I}}\left(c_{L}\right)}{c_{S}-c_{L}}-\tilde{f}_{c \widetilde{T}_{I}}\left(c_{L}\right)\right],
\end{aligned}
$$

where subscripts on $\tilde{f}$ denote partial derivatives, and $\widetilde{m}_{L}$ and $\widetilde{m}_{S}$ are the dimensionless slopes of the liquidus and solidus for the interfacial temperature $\tilde{T}_{I}$.

From the form $\tilde{f}$ we find that the above expressions, when evaluated for the $\alpha-\mathrm{L}$ phase transition are

$$
\begin{aligned}
& \widetilde{m}_{S}^{\alpha L}\left(\tilde{T}_{I}\right)=\frac{\tilde{T}_{I}\left[c_{S}^{\alpha L}-c_{L}^{\alpha L}\right]}{c_{S}^{\alpha} L\left(1-c_{S}^{\alpha L}\right)\left[\tau \tilde{L}_{B} c_{L}^{\alpha L}+\tilde{L}_{A}\left(1-c_{L}^{\alpha L}\right)+I\left(c_{S}\right)-I\left(c_{L}\right)-\left(c_{S}-c_{L}\right) I^{\prime}\left(c_{S}\right)\right]}, \\
& \widetilde{m}_{L}^{\alpha L}\left(\tilde{T}_{I}\right)=\frac{\tilde{T}_{I}\left[c_{S}^{\alpha L}-c_{L}^{\alpha L}\right]}{c_{L}^{\alpha L}\left(1-c_{L}^{\alpha L}\right)\left[\tau \tilde{L}_{B} c_{S}^{\alpha L}+\tilde{L}_{A}\left(1-c_{S}^{\alpha L}\right)+I\left(c_{S}\right)-I\left(c_{L}\right)-\left(c_{S}-c_{L}\right) I^{\prime}\left(c_{L}\right)\right]},
\end{aligned}
$$

and from the symmetry of the phase diagram that $\widetilde{m}_{S}^{\beta L}\left(\widetilde{T}_{I}\right)=-\widetilde{m}_{S}^{\alpha L}\left(\widetilde{T}_{I}\right)$ and $\widetilde{m}_{L}^{\beta L}\left(\widetilde{T}_{I}\right)=$ $-\widetilde{m}_{L}^{\alpha L}\left(\tilde{T}_{I}\right)$.

For the situation in which the interface is curved and moving we may seek the approximation to the relation between the interfacial concentrations and temperature when $\tilde{\sigma}(\tilde{V} / \widetilde{m}+\tilde{K})$ is small. To this end we expand these variables about the equilibrium state, representing the planar stationary interface for the $\alpha-\mathrm{L}$ phase transition, denoted by $c_{S}^{E}, c_{L}^{E}, \tilde{T}^{E}$, i.e. put

$$
c_{S}=c_{S}^{E}+c_{S}^{\prime}, c_{L}=c_{L}^{E}+c_{L}^{\prime}, \widetilde{T}_{I}=\tilde{T}^{E}+\widetilde{T}_{I}^{\prime}, \tilde{\mu}=\tilde{\mu}^{E}+\tilde{\mu}^{\prime}
$$

We linearise the Eq. (104) and Eq. (107), representing the parallel tangent construction in the perturbed (primed) quantities. Eliminating $\tilde{\mu}^{\prime}$ these linearised equations give

$$
\tilde{T}^{\prime}\left[\tilde{f}_{c T}\left(c_{S}^{E}\right)-\frac{\tilde{f}_{T}\left(c_{S}^{E}\right)-\tilde{f}_{T}\left(c_{L}^{E}\right)}{c_{S}^{E}-c_{L}^{E}}\right]=-\tilde{f}_{\mathrm{cc}}\left(c_{S}^{E}\right) c^{\prime}+\frac{\tilde{\sigma}}{c_{S}^{E}-c_{L}^{E}}\left(\frac{\tilde{V}_{n}}{\widetilde{m}}+\tilde{K}\right)
$$


Using the expression for $\widetilde{m}_{S}^{\alpha} L$, Eq. (134) and noting that the factor in square brackets on the right hand side is the same as the denominator in Eq. (132), Eq. (134) gives that

$$
\tilde{T}^{\prime}=\widetilde{m}_{S}^{\alpha L} c_{S}^{\prime}-\frac{\tilde{\sigma}^{\alpha L} \tilde{T}^{E}}{\left[\tau \tilde{L}_{B} c_{L}^{\alpha L}+\tilde{L}_{A}\left(1-c_{L}^{\alpha L}\right)\right]}\left(\frac{\tilde{V}_{n}}{\widetilde{m}}+\tilde{K}\right) .
$$

For the dilute $\alpha-\mathrm{L}$ alloy it is appropriate to consider the limit $c_{S}, c_{L} \rightarrow 0$ and $\tilde{T}^{E} \rightarrow 1$, in which case Eq. (138) can be written in dimensional form as

$$
T_{I}=T_{H}+\left[\frac{\left(k^{\alpha L}-1\right) R T_{H}^{2}}{k^{\alpha L} v_{m} L_{A}}\right] c_{S}-\frac{\sigma^{\alpha L} T_{H}}{L_{A}} K-\frac{V_{n}}{\mu^{\alpha L}}
$$

where all the variables are dimensional in this expression, $k^{\alpha L}$ is the segregation coefficient, defined here as

$$
k^{\alpha L}=\lim _{c_{L} \rightarrow 0} \frac{c_{S}^{\alpha L}}{c_{L}^{\alpha L}}
$$

and $\mu^{\alpha L}$ is the interface mobility coefficient. The analysis can be repeated for the liquid concentration to yield the analogous dimensional expression

$$
T_{I}=T_{H}+\left[\frac{\left(k^{\alpha L}-1\right) R T_{H}^{2}}{v_{m} L_{A}}\right] c_{L}-\frac{\sigma^{\alpha L} T_{H}}{L_{A}} K-\frac{V_{n}}{\mu^{\alpha L}}
$$

for a dilute $\alpha-\mathrm{L}$ alloy. Corresponding expressions can also be obtained for the $\beta-\mathrm{L}$ alloy in a straight forward manner. 


\section{References}

[1] Y. Saito, G. Goldbeck-Wood, and H. Muller-Krumbhaar, Phys. Rev. A. 38, 2148 (1988).

[2] John M. Sullivan, Jr. and Huizi Hao, in Heat Transfer in Melting, Solidification, and Crystal Growth, Vol. 234, ed. Iz S. Habib and Stefan Thynell (ASME, New York, 1993) pp. 14-19.

[3] O. Penrose and P. C. Fife, Physica D 43, 44 (1990).

[4] S-L. Wang, R. F. Sekerka, A. A. Wheeler, B. T. Murray, S. R. Coriell, R. J. Braun, and G. B. McFadden, Physica D 69, 189 (1993).

[5] J. S. Langer, 1978, private communication.

[6] B. I. Halperin, P. C. Hohenberg, and S.-K. Ma, Phys. Rev. B 10 (1974) 139.

[7] J. D. van der Waals, Verhandel. Konink. Akad. Weten. Amsterdam (Sect. 1) Vol. 1, No. 8 (1893).

[8] V. L. Ginzburg and L. D. Landau, Soviet Phys. JETP 20 (1950) 1064.

[9] J. W. Cahn and J. E. Hilliard, J. Chem. Phys. 28 (1958) 258.

[10] G. Caginalp, Arch. Rat. Mech. Anal. 92, 205 (1986).

[11] G. Caginalp, Arch. Rat. Mech. Anal. 92, 205 (1986).

[12] J. B. Collins and H. Levine, Phys. Rev. B 31, 6119 (1985).

[13] G. Caginalp, Phys. Rev. A 39, 5887 (1989).

[14] R. Kobayashi, Physica D 63, 410 (1993).

[15] A. A. Wheeler, B. T. Murray, and R. J. Schaefer, Physica D 66, 243 (1993).

[16] A. A. Wheeler, W. J. Boettinger, and G. B. McFadden, Phys. Rev. A. 45, 7424 (1992).

[17] A. A. Wheeler, W. J. Boettinger, and G. B. McFadden, Phys. Rev. E. 47, 1893 (1993).

[18] James A. Warren and William J. Boettinger, to appear in Acta Metall. et Mater. (1994).

[19] G. Caginalp and W. Xie, Phys. Rev. E. 48, 1897 (1993).

[20] A. Karma, Phase-Field Model of Eutectic Growth, Preprint (1993). 
[21] K. R. Elder, Francois Drolet, J. M. Kosterlitz, and Martin Grant, Phys. Rev. Lett. 72, 677 (1994).

[22] C. Misbah and D. E. Temkin, Phys. Rev. E 49, 3159 (1994).

[23] J. L. Murray, Met. Trans. 15A, 261 (1984).

[24] J. S. Langer, in Directions in Condensed Matter Physics, edited by G. Grinstein and G. Mazenko, pages 164-186, World Science Publishers, 1986.

[25] R. Kobayashi, Bull. Jpn. Soc. Ind. Appl. Math. 1, 22 (1991).

[26] J. E. Hilliard, Spinodal Decomposition, pages 497-560, American Society For Metals, Metals Park, Ohio, USA, 1970.

[27] F. Larché and J. W. Cahn, Acta Met. 26, 1579 (1978).

[28] H. E. Cook and J. E. Hilliard, J. Appl. Phys. 40, 2191 (1969).

[29] T. Tsakalakos, Acta Met. 33, 1939 (1985).

[30] P. Dugan and T. Tsakalakos, Mat. Sci. and Eng., 171 (1990).

[31] G. B. McFadden, A. A. Wheeler, R. J. Braun, S. R. Coriell, and R. F. Sekerka, Phys. Rev. E 48, 2016 (1993).

[32] S. M. Allen and J. W. Cahn, Acta Metall. 27, 1085 (1979).

[33] L. Bronsard and F. Reitich, On the three-phase boundary motion and the singular limit of a vector- valued ginzburg-landau equation., Technical Report 92-NA-022, Center for Nonlinear Analysis, Carnegie Mellon University, 1992.

[34] T. C. Oppe, W. D. Joubert, and D. R. Kincaid, NSPCG user guide (version 1.0), Technical Report CNA-216, Center of Numerical Analysis, University of Texas at Austin, 1988. 


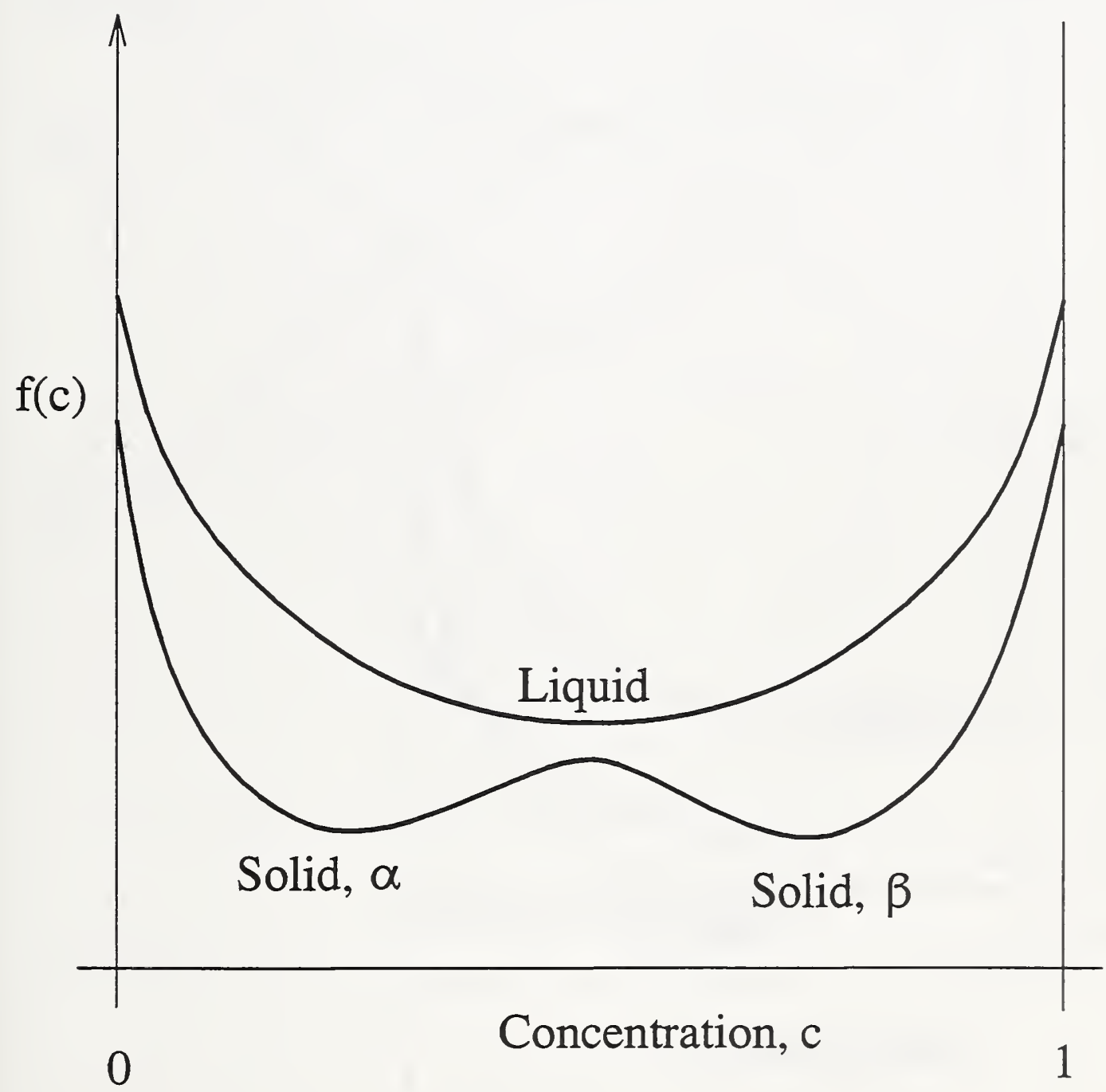

Figure 1: A schematic plot of the bulk free energy densities $f_{L}(c, T)$ and $f_{S}(c, T)$ of the liquid and solid phases versus $c$ for Model I at a temperature below the eutectic temperature; the curves are symmetric about $c=1 / 2$. 


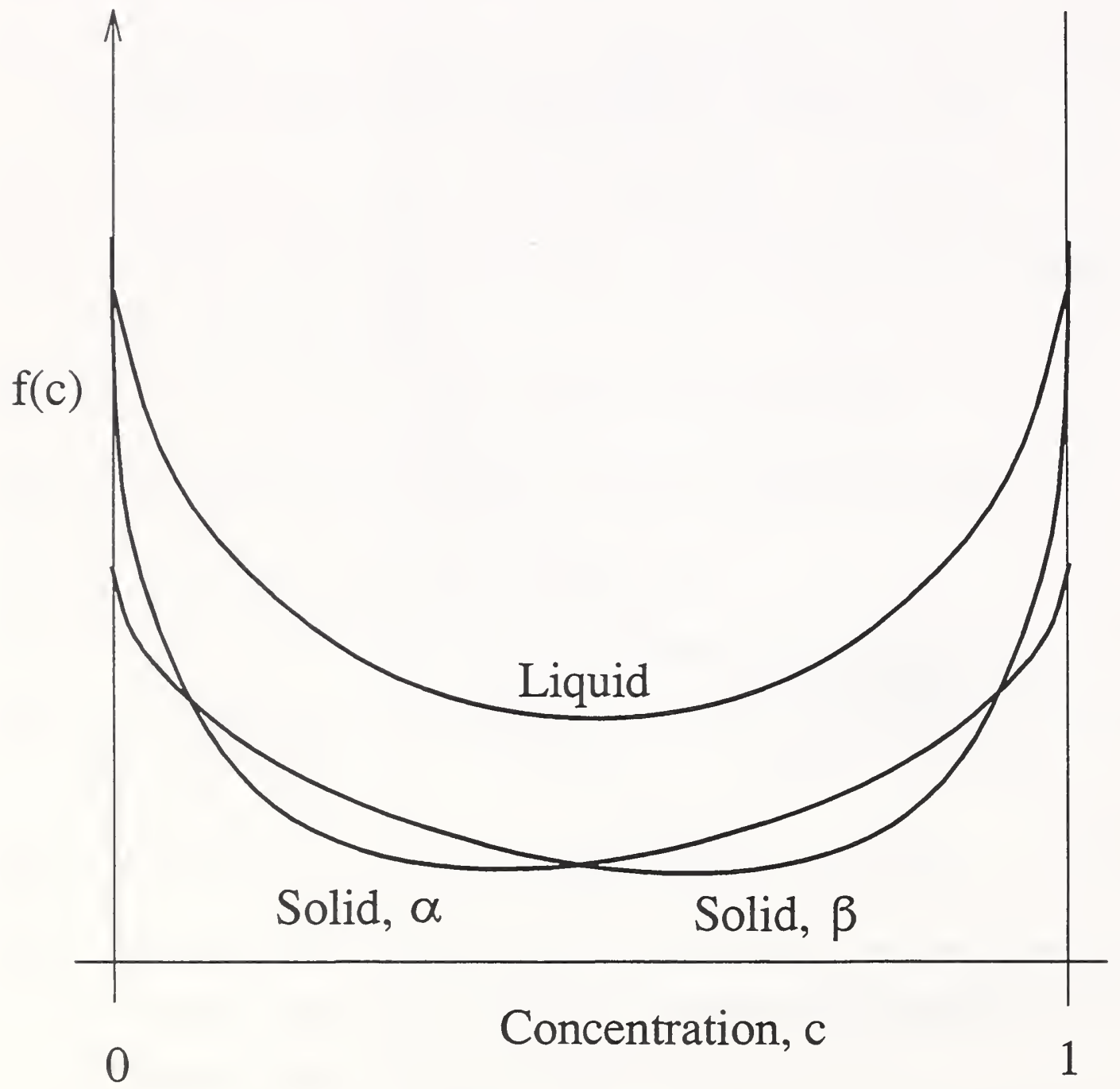

Figure 2: A schematic plot of the bulk free energy densities $f_{\alpha}(c, T)$ and $f_{\beta}(c, T)$ of the two solid phases and $f_{L}(c, T)$ of the liquid phase versus $c$ for Model II at a temperature below the eutectic temperature. 


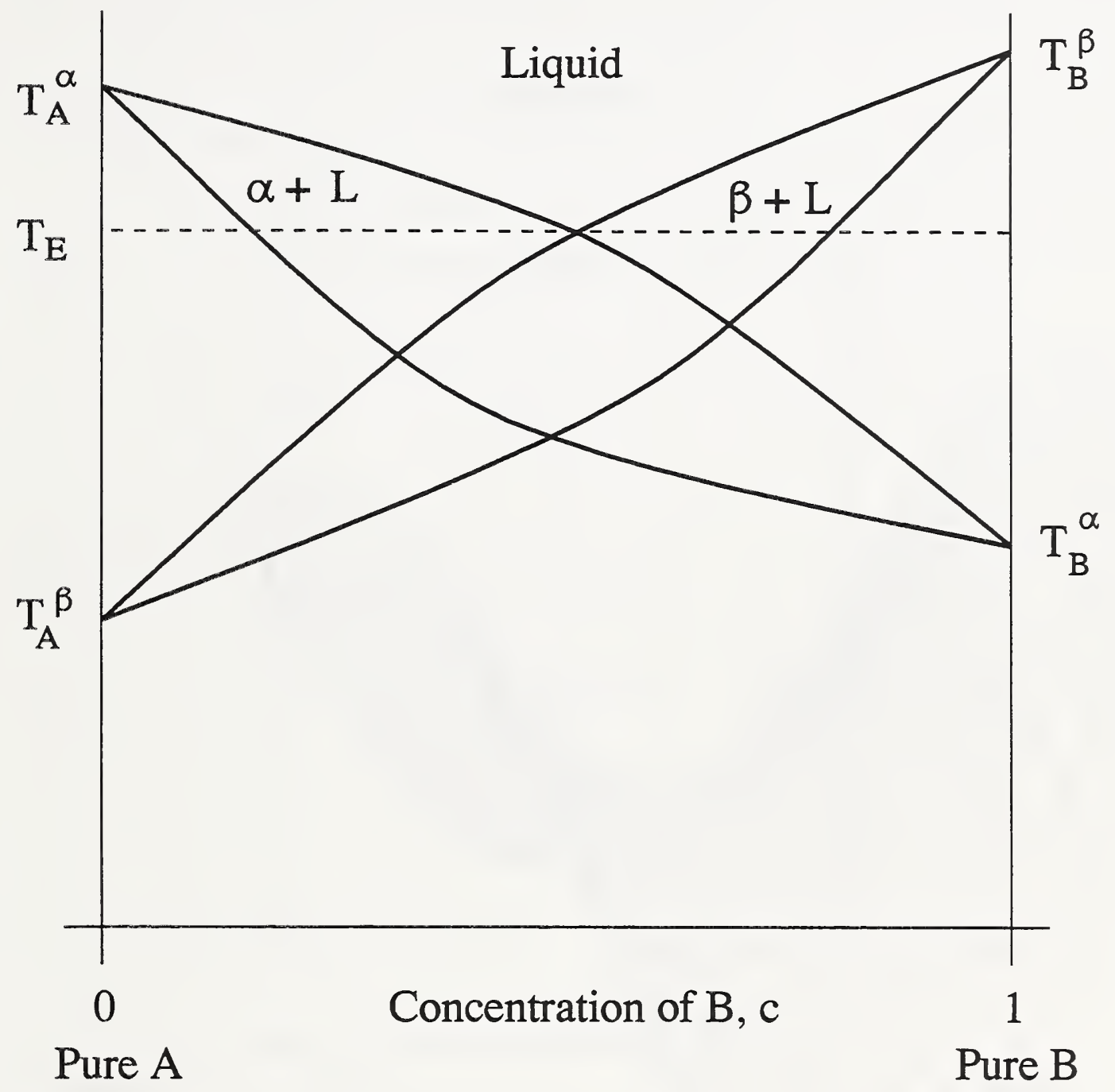

Figure 3: A schematic drawing of superimposed liquid/ $\alpha$ and liquid/ $\beta$ phase diagrams. The eutectic temperature is $T_{E}$ and the phase boundaries below this temperature are metastable with respect to the formation of $\alpha+\beta$ mixture. 


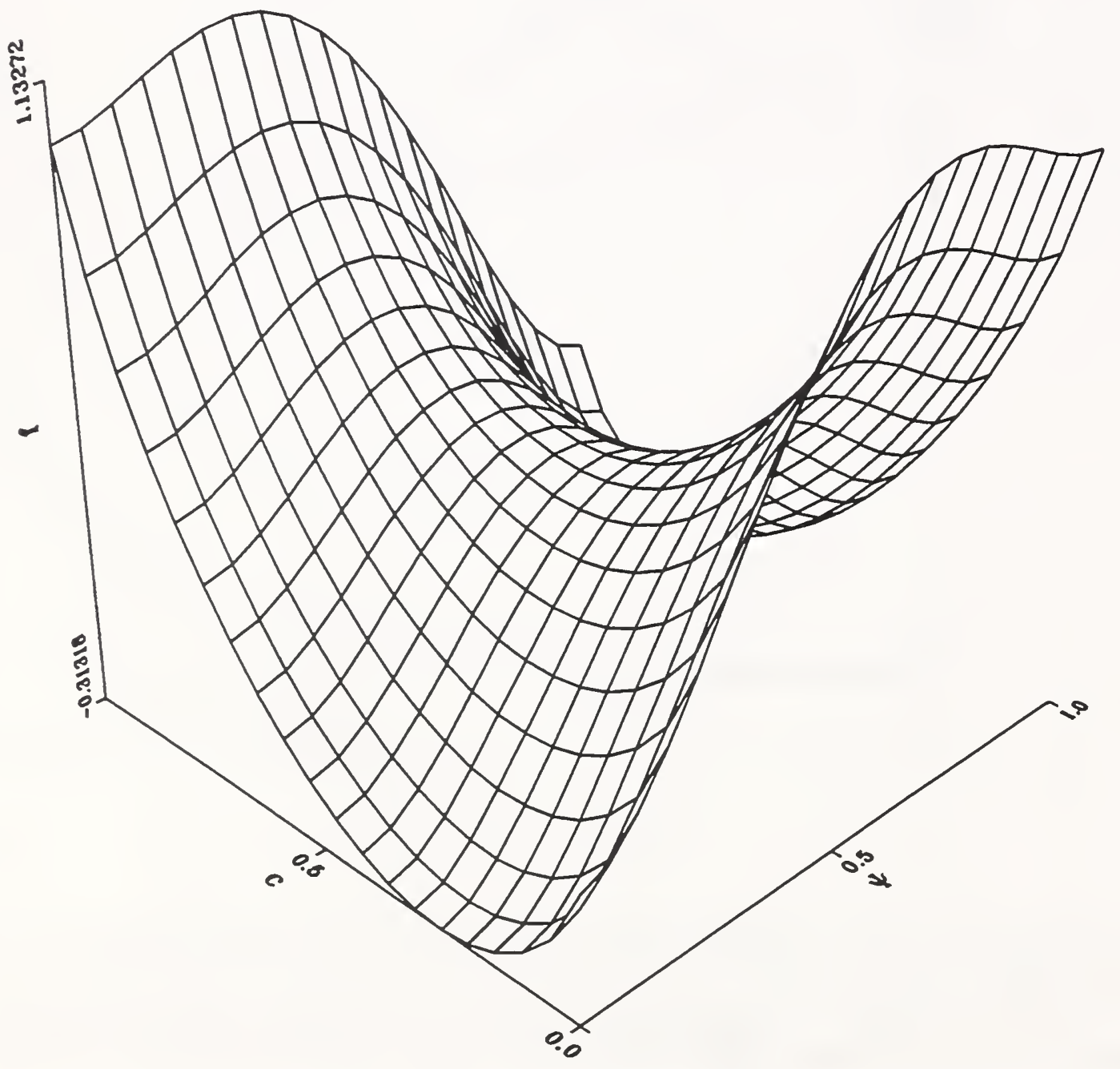

Figure 4: A plot of $f$ as a function of $\psi$ and $c$ in the solid $(\phi=1)$ for Model II. 


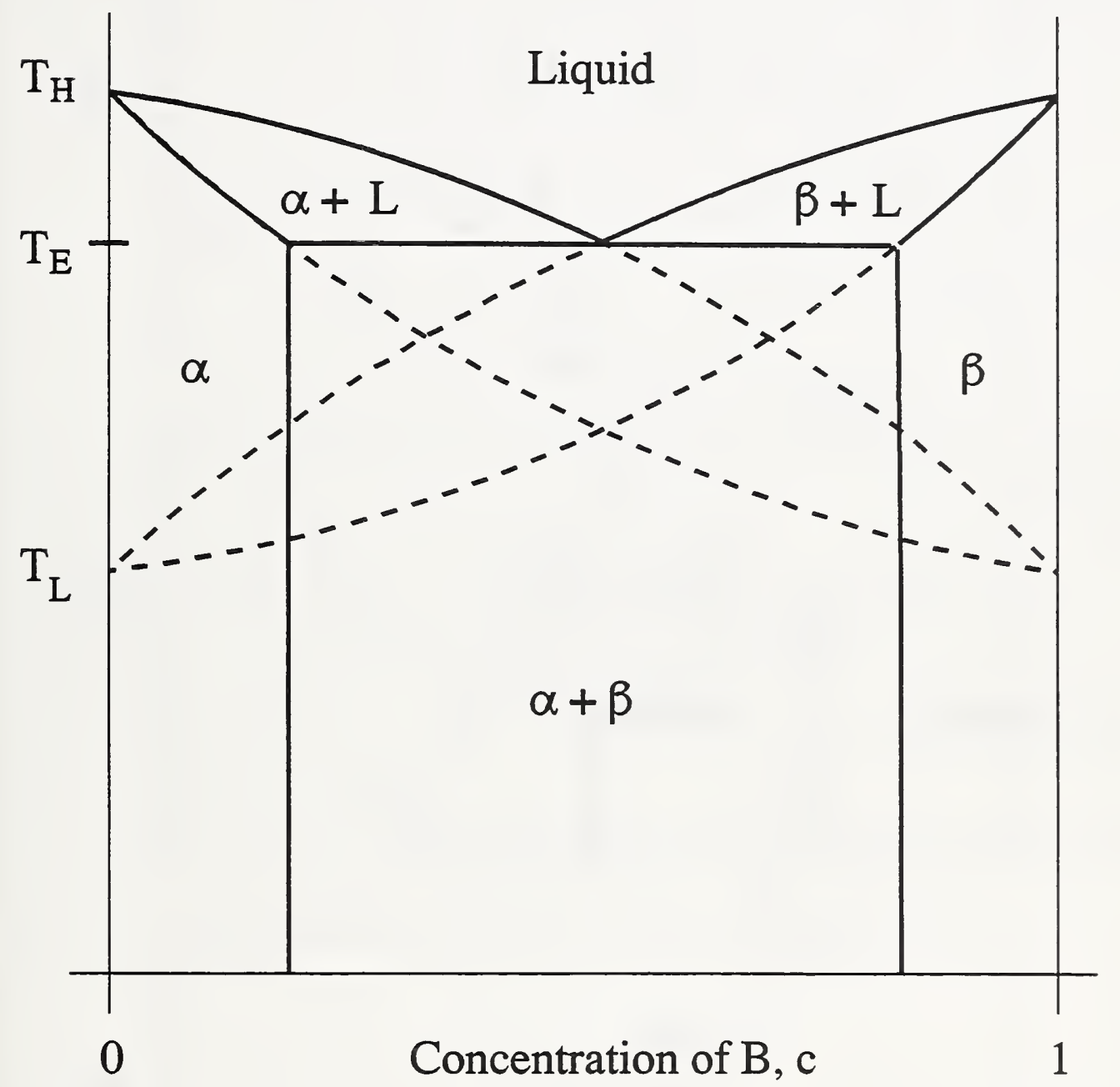

Pure A

Pure B

Figure 5: A schematic drawing of the phase diagram for the symmetric alloy. The vertical solvus curves are indicated by the dashed lines. 


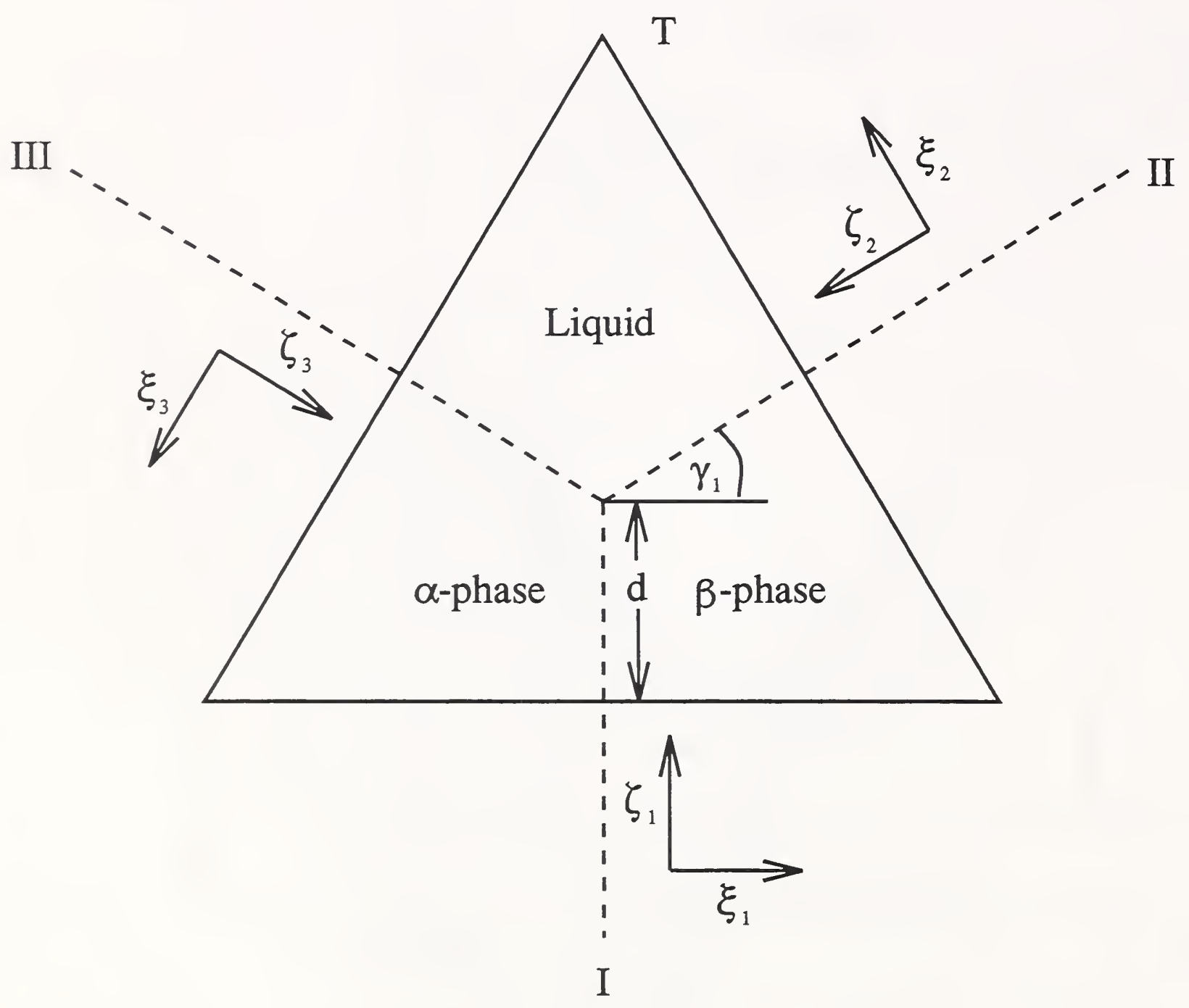

Figure 6: Schematic diagram of the trijunction. 

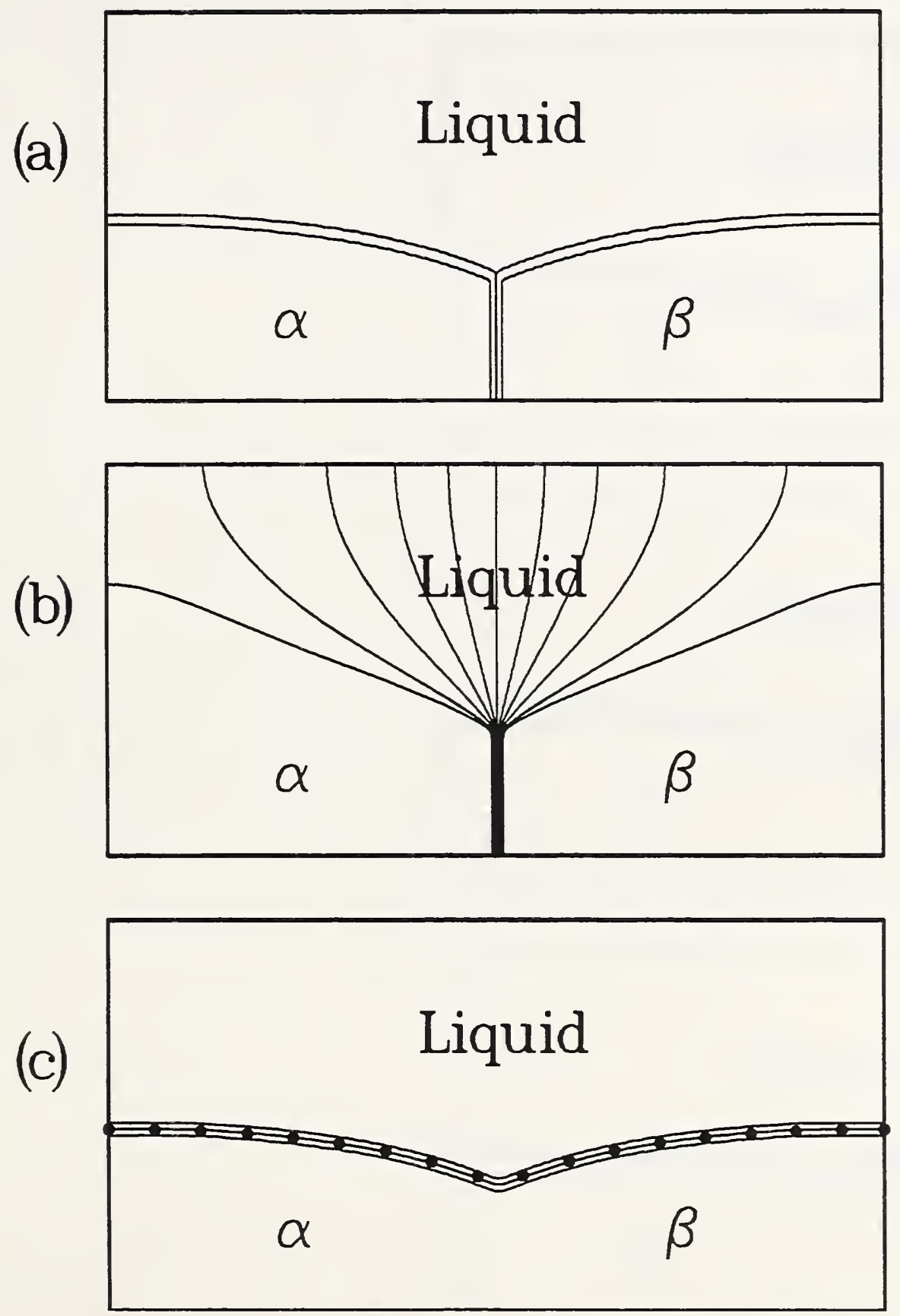

Figure 7: The results of our computations for the the dimensionless parameters given in Table 1. (a) shows the concentration field with contours at $5 \%, 50 \%$ and $95 \%$ of the range of concentrations, (b) show the $\psi$ phase-field with contours at $\psi=0.05,0.1,0.2, \ldots 0.9,0.95$. and (c) the $\phi$ phase field with contours at $\psi=0.05,0.5$ and 0.95 . The solid circles represent the position of the solid/liquid interface given from the sharp interface solution. 
(a)

\section{Liquid}

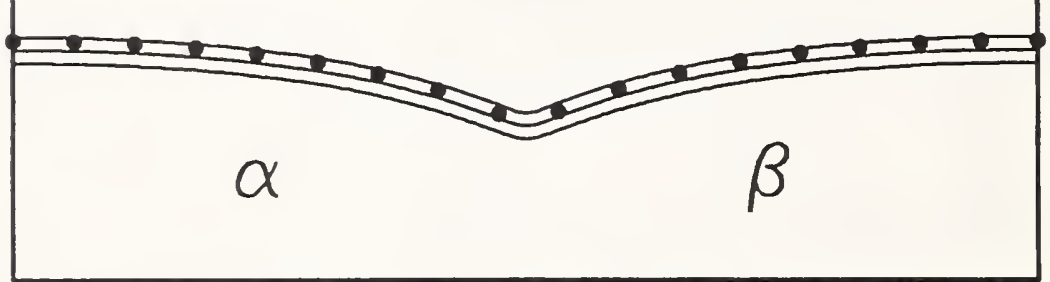

(b)

\section{Liquid}

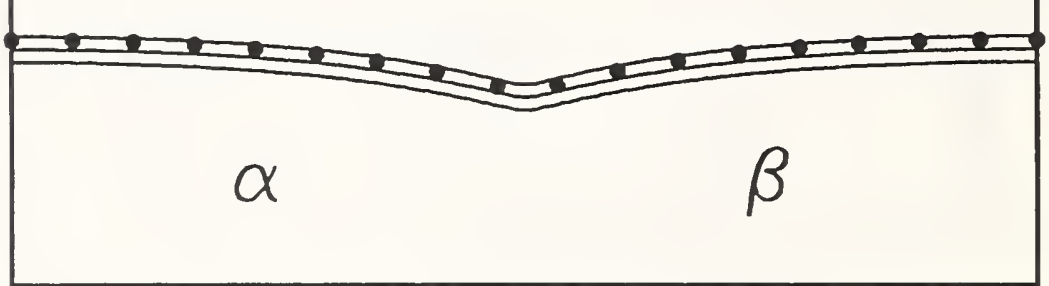

(c)

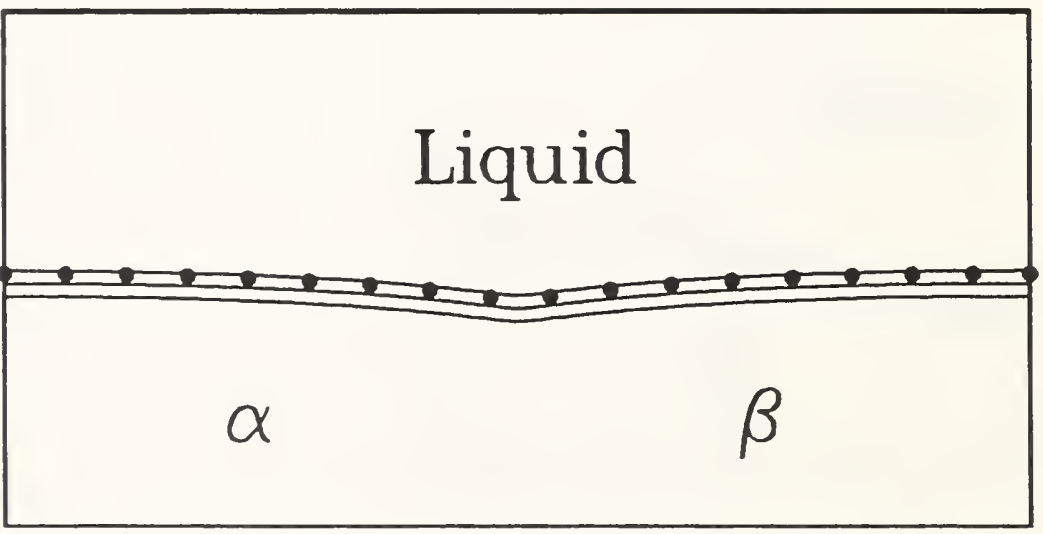

Figure 8: The $\phi$ phase-field from our computations for the the dimensionless parameters given in Table 1, but with $\tilde{\epsilon}_{\phi}=\tilde{\epsilon}_{\psi}=0.1, \tilde{G}=1 \tilde{L}=1$ and three different values of the solid surface energy; (a) $\tilde{\sigma}_{S}=0.1871$, (b) $\tilde{\sigma}_{S}=0.1198$, (c) $\tilde{\sigma}_{S}=0.06132$. The solid circles represent the position of the solid/liquid interface given from the sharp interface solution. 

